\title{
ALGEBROID NATURE \\ OF THE CHARACTERISTIC CLASSES OF FLAT BUNDLES
}

\author{
JAN KUBARSKI \\ Institute of Mathematics, Technical University of Łódź \\ 90-924 Eódź, Al. Politechniki 11, Poland \\ E-mail: kubarski@ck-sg.p.lodz.pl
}

\begin{abstract}
The following two homotopic notions are important in many domains of differential geometry:

- homotopic homomorphisms between principal bundles (and between other objects),

- homotopic subbundles.

They play a role, for example, in many fundamental problems of characteristic classes. It turns out that both these notions can be - in a natural way - expressed in the language of Lie algebroids. Moreover, the characteristic homomorphisms of principal bundles (the ChernWeil homomorphism [K4], or the subject of this paper, the characteristic homomorphism for flat bundles) are invariants of Lie algebroids of these bundles. This enables one to construct the characteristic homomorphism of a flat regular Lie algebroid, measuring the incompatibility of the flat structure with a given subalgebroid. For two given Lie subalgebroids, these homomorphisms are equivalent if the Lie subalgebroids are homotopic. Some new examples of applications of this characteristic homomorphism to a transitive case (for TC-foliations) and to a non-transitive case (for a principal bundle equipped with a partial flat connection) are pointed out (Ex. 3.1). An example of a transitive Lie algebroid of a TC-foliation which leads to the nontrivial characteristic homomorphism is obtained.
\end{abstract}

\section{Preliminaries}

1.1. Characteristic homomorphism of flat bundles. Consider

- a $G$-principal bundle $P$,

- a flat connection $\omega$ in $P$,

- an $H$-reduction $P^{\prime} \subset P, H$ being a closed Lie subgroup of $G$.

Let $\mathfrak{h}$ and $\mathfrak{g}$ be the Lie algebras of $H$ and $G$, respectively. There is a characteristic

1991 Mathematics Subject Classification: Primary 57R20; Secondary 53C12, 55R40.

The paper is in final form and no version of it will be published elsewhere. 
homomorphism

$$
\Delta_{\# P, P^{\prime}, \omega}: H^{\star}(\mathfrak{g}, H) \longrightarrow H_{d R}(M)
$$

measuring the independence of $\omega$ and $P^{\prime}$, which means to what extent $\omega$ is not contained in $T P^{\prime}$ (for details, see for example [K-T]). One of the fundamental properties of $\Delta_{\# P, P^{\prime}, \omega}$ is the independence of the choice of a homotopic subbundle $P^{\prime}$ :

$$
\text { if } P^{\prime} \stackrel{\text { homotopic }}{\approx} P^{\prime \prime} \subset P \text {, then } \Delta_{\# P, P^{\prime}, \omega}=\Delta_{\# P, P^{\prime \prime}, \omega} \text {. }
$$

This means that the nontriviality of $\Delta_{\# P, P^{\prime}}$ implies the impossibility of the homotopic changing of $P^{\prime}$ to contain the connection $\omega$.

We recall that the domain $H^{\star}(\mathfrak{g}, H)$, called the relative Lie algebra cohomology [K-T], is the cohomology space of the complex $\left(\bigwedge(\mathfrak{g} / \mathfrak{h})_{I}^{\star}, d^{H}\right)$,

$$
H^{\star}(\mathfrak{g}, H)=H^{\star}\left(\bigwedge(\mathfrak{g} / \mathfrak{h})_{I}^{\star}, d^{H}\right)
$$

where $\Lambda(\mathfrak{g} / \mathfrak{h})_{I}^{\star}$ is the space of invariant elements (with respect to the adjoint representation), whereas the differential $d^{H}$ is defined in such a way that, for $\psi \in \bigwedge^{k}(\mathfrak{g} / \mathfrak{h})_{I}^{\star}$ and $w_{i} \in \mathfrak{g}$,

$$
\left\langle d^{H}(\psi),\left[w_{i}\right] \wedge \ldots \wedge\left[w_{k}\right]\right\rangle=\sum_{i<j}(-1)^{i+j}\left\langle\psi,\left[\left[w_{i}, w_{j}\right]\right] \wedge\left[w_{i}\right] \wedge \ldots \hat{i} \ldots \hat{j} \ldots \wedge\left[w_{k}\right]\right\rangle
$$

$\left(\left[w_{i}, w_{j}\right]\right.$ is the bracket in the Lie algebra $\mathfrak{g}$, whereas $[w]$ denotes the equivalence class in $\mathfrak{g} / \mathfrak{h})$. The homomorphism $\Delta_{\# P, P^{\prime, \omega}}$ on the level of forms $\Delta_{\star}: \Lambda(\mathfrak{g} / \mathfrak{h})^{\star} \rightarrow \Omega(M)$ can be defined as follows: $\Delta_{\star}(\psi)$ for $\psi \in \Lambda^{k}(\mathfrak{g} / \mathfrak{h})^{\star}$ is the only $k$-form for which

$$
\pi^{\prime \star}\left(\Delta_{\star} \psi\right)=\frac{1}{k !} \cdot i^{\prime \star}\left\langle\bigwedge^{k} s^{\star}(\psi), \omega \wedge \ldots \wedge \omega\right\rangle,
$$

with $i^{\prime}: P^{\prime} \hookrightarrow P, s: \mathfrak{g} \rightarrow \mathfrak{g} / \mathfrak{h}, \pi^{\prime}: P^{\prime} \rightarrow M, \omega \wedge \ldots \wedge \omega \in \Omega^{k}\left(P ; \wedge^{k} \mathfrak{g}\right)$.

It turns out that the characteristic homomorphism of a flat bundle described above is a notion of its Lie algebroid. A construction of its version on the category of regular Lie algebroids is the aim of this work. A simple example of a transitive Lie algebroid of the TC-foliation of left cosets of a nonclosed Lie subgroup in a Lie group having this homomorphism nontrivial is given (see Chap. 8).

1.2. The category of Lie algebroids. Differential geometry has discovered many objects which determine Lie algebroids playing a role analogous to that of Lie algebras for Lie groups. For example: differential groupoids, principal bundles, vector bundles, actions of Lie groups on manifolds, transversally complete foliations, nonclosed Lie subgroups, Poisson manifolds, some complete closed pseudogroups, etc. The category of Lie algebroids is more elastic than the category of principal bundles. It enables one to generalize the characteristic homomorphisms in the direction of nontransitive objects.

1.2.1. Definitions and notations. By a Lie algebroid on a manifold $M$ [P1], [P2], we mean a system $A=(A, \llbracket \cdot, \cdot \rrbracket, \gamma)$ consisting of a vector bundle $A$ on $M$ and mappings $\llbracket \cdot, \rrbracket: \operatorname{Sec} A \times \operatorname{Sec} A \rightarrow \operatorname{Sec} A, \gamma: A \rightarrow T M$, such that (1) $(\operatorname{Sec} A, \llbracket \cdot, \cdot \rrbracket)$ is an $\mathbb{R}$-Lie algebra, (2) $\gamma$, called the anchor, is a homomorphism of vector bundles, (3) $\operatorname{Sec} \gamma: \operatorname{Sec} A \rightarrow \mathfrak{X}(M)$, $\xi \mapsto \gamma \circ \xi$, is a homomorphism of Lie algebras, (4) $\llbracket \xi, f \cdot \eta \rrbracket=f \cdot \llbracket \xi, \eta \rrbracket+(\gamma \circ \xi)(f) \cdot \eta$, $f \in C^{\infty}(M)$. A Lie algebroid $A$ is said to be transitive if $\gamma$ is an epimorphism of vector 
bundles, and regular if $\gamma$ is of constant rank. In the last situation, if $\operatorname{Im} \gamma=F \subset T M$, then $F$ is an involutive distribution and $A$ is called a regular Lie algebroid over the foliated manifold $(M, F)$. In the sequel, we adopt the notions and the notations from [P1], [M1], [K4], among them, the adjoint bundle of Lie algebras $\boldsymbol{g}:=\operatorname{Ker} \gamma$, the Atiyah sequence $0 \rightarrow \boldsymbol{g} \rightarrow A \rightarrow F \rightarrow 0$ and the notion of a connection.

A principal bundle $P$ and a TC-foliation $(M, E)$ determine transitive Lie algebroids $A(P)[\mathrm{M} 1],[\mathrm{K} 3],[\mathrm{K} 8]$ and $A(M, E)$ [MO1], respectively. A geometrically important object consisting of a given principal bundle $P$ on $M$ and a foliation $F$ on the base $M$ of $P$ is a source of a nontransitive Lie algebroid $A(P)^{F}$ on a foliated manifold $(M, F)$ for which $A(P)^{F}=\gamma^{-1}[F], \gamma: A(P) \rightarrow T M$ being the anchor of $A(P)$. Poisson manifolds are sources of nontransitive Lie algebroids [C-D-W].

TheOrem 1.1 [K2, Th. 1.1]. If $A^{\prime} \subset A(P)$ is a transitive Lie subalgebroid of the Lie algebroid $A(P)$ of a principal bundle $P=(P, \pi, M, G, \cdot)$, then there exists a reduction $P^{\prime}$ of $P$ having $A^{\prime}$ as its Lie algebroid.

1.2.2. The Lie algebroid of a vector bundle, representations and invariant crosssections. With each vector bundle $\mathfrak{f}$ we associate a transitive Lie algebroid $A(\mathfrak{f})$ which is the Lie algebroid of the principal bundle $L(\mathfrak{f})$ of all frames of $\mathfrak{f}[\mathrm{M} 1]$ or of the Lie groupoid $G L(\mathfrak{f})$ of all linear isomorphisms between fibres, see for example [N-V-Q], [KU], [M1].

Equivalently we can construct this Lie algebroid in such a way that the fibre $A(\mathfrak{f})_{\mid x}$ over $x \in M$ is equal to the space of all linear homomorphisms

$$
l: \operatorname{Sec} \mathfrak{f} \longrightarrow \mathfrak{f}_{\mid x}
$$

for which there exists a vector $u \in T_{x} M$ such that $l(f \cdot \nu)=f(x) \cdot l(\nu)+u(f) \cdot \nu(x)$, $f \in C^{\infty}(M), \nu \in \operatorname{Sec} \mathfrak{f}$ (see [K4], [B]). The space of global cross-sections $\operatorname{Sec} A(\mathfrak{f}$ ) is equal to the space of all covariant differential operators in $\mathfrak{f}[\mathrm{M} 1]$.

By a representation of $A$ on $\mathfrak{f}$ (both over a manifold $M$ ) we mean a strong homomorphism of regular Lie algebroids $T: A \rightarrow A(\mathfrak{f})$ [M1]. For a cross-section $\xi \in \operatorname{Sec} A$, its image $T \circ \xi$ is a cross-section of $A(\mathfrak{f})$, therefore it determines a covariant differential operator $\mathfrak{L}_{T \circ \xi}: \operatorname{Sec} \mathfrak{f} \rightarrow \operatorname{Sec} \mathfrak{f}$. A cross-section $\nu \in \operatorname{Sec} \mathfrak{f}$ is called $T$-invariant if $\mathfrak{L}_{T \circ \xi}(\nu)=0$ for all $\xi \in \operatorname{Sec} A$. The space of all $T$-invariant cross-sections of $\mathfrak{f}$ is denoted by $(\operatorname{Sec} \mathfrak{f})_{I^{0}(T)}$ or, briefly, by $(\operatorname{Sec} \mathfrak{f})_{I^{0}}$.

ExAmple 1.2. (1) The adjoint representation $\operatorname{ad}_{A}: A \rightarrow A(\boldsymbol{g})$ of $A$ on its adjoint bundle of Lie algebras $\boldsymbol{g}$ is defined in such a way that for $\xi \in \operatorname{Sec} A$,

$$
\mathfrak{L}_{\operatorname{ad}_{A} \circ \xi}(\nu)=\llbracket \xi, \nu \rrbracket, \quad \nu \in \operatorname{Sec} \boldsymbol{g} .
$$

(2) A given representation $T: A \rightarrow A(\mathfrak{f})$ yields the representations on the associated vector bundles $\mathfrak{f}^{\star}, \bigwedge^{k} \mathfrak{f}, \bigvee^{k} \mathfrak{f}$, etc. [K4], denoted also by $T$.

In the context of transitive Lie algebroids, we have two fundamental facts:

- Two cross-sections of $\mathfrak{f}$ invariant with respect to a representation $T$ of $A$ on $\mathfrak{f}$, equal at one point, must be equal globally [M1], [K9]. (Remark: therefore, for a regular Lie algebroid over a foliated manifold $(M, F)$ these cross-sections are equal on the whole leaf of the foliation $F$, passing through this point.) 
- Th. IV.1.19 of [M1, p.195] gives the homotopic description of the space $(\operatorname{Sec} \mathfrak{f})_{I^{o}(M)}$ of all invariant cross-sections with respect to a representation of a transitive Lie algebroid.

1.2.3. The Chern-Weil homomorphism. In [K4] the Chern-Weil homomorphism $d_{A}$ : $I_{A} \rightarrow H_{F}(M)$ of a regular Lie algebroid $A$ over $(M, F)$ is constructed, whose domain $I_{A}$ is the algebra of $\operatorname{ad}_{A^{-}}$-invariant cross-sections of $\bigvee^{k} \boldsymbol{g}^{\star}, k \in \mathbb{N}$. For an integrable Lie algebroid $A=A(P), P$ being a connected principal bundle (the structure Lie group $G$ may be disconnected!), this algebra is isomorphic to the space of invariant polynomials $\left(\bigvee \mathfrak{g}^{\star}\right)_{I}$. Note that this holds although in the Lie algebroid $A(P)$ there is no direct information about the structure Lie group of $P$ which may be disconnected and that a Lie algebroid is - in some sense - a simpler structure than a principal bundle (nonisomorphic principal bundles can possess isomorphic Lie algebroids, see [K3]). The case $A=A(P)^{F}$ is considered in $[\mathrm{K} 6]$. Here, $I_{A}$ contains elements of the form $\sum f^{i} \cdot \Gamma_{i}, f^{i}$ being $F$-basic functions and $\Gamma_{i} \in I_{A(P)}$, i.e. contains the subalgebra $\mathcal{F}_{b}(M, F) \cdot I_{A(P)}$. In $[\mathrm{K} 6]$ there is an example of $P$ and $F$ for which in the domain $I_{A(P)^{F}}$ there are elements not belonging to $\mathcal{F}_{b}(M, F) \cdot I_{A(P)}$, called singular. For the principal bundle $P$ of frames of a vector bundle $\mathfrak{f}$ and any foliation $F$ on the base $M$, the restriction $h_{A(P)}$ to $\mathcal{F}_{b}(M, F) \cdot I_{A(P)}$ agrees with the construction of Moore-Schochet [M-S] of the characteristic homomorphism of a vector bundle $\mathfrak{f}$ over a foliated manifold. Therefore $h_{A(P)^{F}}$ can be subtler than the one constructed in [M-S].

For the Lie algebroid $A(G ; H)$ over $G / \bar{H}$ of the TC-foliation of left cosets of a nonclosed Lie subgroup $H$ in a connected Lie group $G[\mathrm{~K} 4]$, the domain $I_{A(G ; H)}$ of its Chern-Weil homomorphism is isomorphic to $\bigvee(\overline{\mathfrak{h}} / \mathfrak{h})^{\star}, \mathfrak{h}, \overline{\mathfrak{h}}$ being the Lie algebras of $H$ and its closure $\bar{H}$. For any connected, compact and semisimple Lie group $G$, we have that $h_{A(G ; H)}^{2}:(\overline{\mathfrak{h}} / \mathfrak{h})^{\star} \rightarrow H^{2}(G / \bar{H})$ is a monomorphism; assuming (in addition) the simple connectedness of $G$, we obtain - according to the Almeida-Molino theorem $[\mathrm{A}-\mathrm{M}]$ - a nonintegrable transitive Lie algebroid having the nontrivial Chern-Weil homomorphism.

1.2.4. Invariant cross-sections over $\mathbb{R} \times M$. In [K4, Defs.1.1.5 and 2.3.1] there are notions of the inverse-image of a regular Lie algebroid $f^{\wedge} A$ and the inverse-image of a representation $f^{\star} T$ via a morphism of foliated manifolds $f:\left(M^{\prime}, E^{\prime}\right) \rightarrow(M, E)$, where $A$ is any regular Lie algebroid over $(M, E)$.

According to [K4, Th.2.4.4], the linear mapping $f^{\star}: \operatorname{Sec} \mathfrak{f} \rightarrow \operatorname{Sec} f^{\star} \mathfrak{f}, \nu \mapsto \nu \circ f$, can be restricted to the space of cross-sections invariant under $T$ and $f^{\star} T$, respectively:

$$
f_{I^{0}}^{\star}:(\operatorname{Sec} \mathfrak{f})_{I^{0}(T)} \longrightarrow\left(\operatorname{Sec} f^{\star} \mathfrak{f}\right)_{I^{0}\left(f^{\star} T\right)} .
$$

The following theorem plays a crucial role in all problems of the homotopic independence of the characteristic homomorphisms considered on the category of Lie algebroids.

TheOREM 1.3 (on invariant cross-sections over $\mathbb{R} \times M,[\mathrm{~K} 9]$ ). Let $B$ be a regular Lie algebroid over the foliated manifold $(\mathbb{R} \times M, T \mathbb{R} \times E)$ and $\mathfrak{f}$ a vector bundle over $\mathbb{R} \times M$, and $T: A \rightarrow A(\mathfrak{f})$ a given representation. Take $t \in \mathbb{R}$ and the mapping $f_{t}: M \rightarrow \mathbb{R} \times M$, $x \mapsto(t, x)$. Then the restriction mapping $f_{t}^{\star}: \operatorname{Sec} \mathfrak{f} \rightarrow \operatorname{Sec} \mathfrak{f}_{\mid\{t\} \times M}\left(\mathfrak{f}_{\mid\{t\} \times M} \cong f_{t}^{\star} \mathfrak{f}\right)$ maps isomorphically the space of invariant cross-sections with respect to $T$ onto the space of 
invariant cross-sections with respect to the restricted representation $f_{t}^{\star} T$ :

$$
\left(f_{t}^{\star}\right)_{I^{0}}:(\operatorname{Sec} \mathfrak{f})_{I^{0}(T)} \stackrel{\cong}{\longrightarrow}\left(\operatorname{Sec} \mathfrak{f}_{\mid\{t\} \times M}\right)_{I^{0}\left(f_{t}^{\star} T\right)} .
$$

1.2.5. Cohomology with coefficients. Let $A$ and $\mathfrak{f}$ be a Lie algebroid and a vector bundle, both over the same manifold, say $M$. Each element of

$$
\Omega_{A}(M ; \mathfrak{f})=\bigoplus_{q \geq 0} \Omega_{A}^{q}(M ; \mathfrak{f}),
$$

where $\Omega_{A}^{q}(M ; \mathfrak{f})=\operatorname{Sec}\left(\bigwedge^{q} A^{\star} \otimes \mathfrak{f}\right)$, will be called an A-differential form of degree $q$, with values in $\mathfrak{f}$; while, for the trivial vector bundle $\mathfrak{f}=M \times \mathbb{R}$, briefly: an A-differential form of degree $q$ (or a real form). In the case $A=T M$ (the usual differential forms on $M$ ), the space of $A$-differential forms with values in $\mathfrak{f}$ (analogously, the space of real forms) will be denoted by $\Omega(M ; \mathfrak{f})\left(\Omega(M)\right.$, respectively). For an involutive $C^{\infty}$ constant dimensional distribution $E$ on $M, \Omega_{E}(M ; \mathfrak{f})$ consists of the so-called tangential differential forms on the foliated manifold $(M, E)[\mathrm{M}-\mathrm{S}],[\mathrm{K} 4]$.

$\Omega_{A}(M ; \mathfrak{f})$ is a graded module over $\Omega^{0}(M)=C^{\infty}(M)$ and a module over the algebra $\Omega_{A}(M)$ of real forms.

Let $\mathfrak{f}^{1}, \ldots, \mathfrak{f}^{k}, \mathfrak{f}$ be vector bundles over $M$. An arbitrary $k$-linear homomorphism of vector bundles $\varphi: \mathfrak{f}^{1} \times \ldots \times \mathfrak{f}^{k} \rightarrow \mathfrak{f}$ determines the mapping

$$
\varphi_{\star}: \Omega_{A}\left(M ; \mathfrak{f}^{1}\right) \times \ldots \times \Omega_{A}\left(M ; \mathfrak{f}^{k}\right) \longrightarrow \Omega_{A}(M ; \mathfrak{f})
$$

defined by the standard formula

$$
\begin{aligned}
& \varphi_{\star}\left(\Psi_{1}, \ldots, \Psi_{k}\right)\left(\xi_{1}, \ldots, \xi_{m}\right) \\
= & \frac{1}{q_{1} ! \cdot \ldots \cdot q_{k} !} \cdot \sum_{\sigma} \operatorname{sgn} \sigma \cdot \varphi\left(\Psi_{1}\left(\xi_{\sigma(1)}, \ldots\right), \ldots, \Psi_{k}\left(\ldots, \xi_{\sigma(m)}\right)\right)
\end{aligned}
$$

in which $m=\sum q_{i}, q_{i}=$ the degree of $\Psi_{i}$.

For a given representation $T: A \rightarrow A(\mathfrak{f})$ of a Lie algebroid $A$ on a vector bundle $\mathfrak{f}$, we have three operators ([MR], [K1], [M1])

$$
\iota_{\xi}, \Theta_{\xi}^{T}, d^{T}: \Omega_{A}(M ; \mathfrak{f}) \longrightarrow \Omega_{A}(M ; \mathfrak{f}), \quad \xi \in \operatorname{Sec} A,
$$

called the substitution operator, the Lie derivative (with respect to $\xi$ ), and the exterior derivative.

For real forms - considering the trivial representation $T: A \rightarrow A(M \times \mathbb{R})=T M \times$ $\operatorname{End}(\mathbb{R})$ defined by $\mathfrak{L}_{T \circ \xi}(\mathfrak{f})=(\gamma \circ \xi)(\mathfrak{f})$ for $f \in \Omega^{0}(M)$ and $\xi \in \operatorname{Sec} A$ or, equivalently, by $T(v)=(\gamma(v), 0))$ - the operator $d^{T}$ will be denoted by $d^{A}$. In particular, if $A=E \subset T M$ is an involutive constant rank distribution on $M$, we obtain the standard operator of the exterior derivative $d^{E}$ of tangential differential forms [M-S].

For arbitrary vector bundles $\mathfrak{f}^{1}, \ldots, \mathfrak{f}^{k}, \mathfrak{f}$ over $M$ and a $k$-linear homomorphism $\varphi$ : $\mathfrak{f}^{1} \times \ldots \times \mathfrak{f}^{k} \rightarrow \mathfrak{f}$ and forms $\Psi_{j} \in \Omega_{A}^{q_{j}}\left(M ; \mathfrak{f}^{j}\right)$, we have

$$
\iota_{\xi}\left(\varphi_{\star}\left(\Psi_{1}, \ldots, \Psi_{k}\right)\right)=\sum_{j=1}^{k}(-1)^{q_{1}+\cdots+q_{j-1}} \varphi_{\star}\left(\Psi_{1}, \ldots, \iota_{\xi} \Psi_{j}, \ldots, \Psi_{k}\right) .
$$

Let now $T^{1}, \ldots, T^{k}, T$ denote fixed representations of $A$ on $\mathfrak{f}^{1}, \ldots, \mathfrak{f}^{k}, \mathfrak{f}$, respectively, and assume that $\varphi$ is Hom-invariant where Hom denotes the induced representation on the 
space of $k$-linear homomorphisms $\operatorname{Hom}^{k}\left(T^{1}, \ldots, T^{k} ; T\right)$ defined by the formula

$$
\mathfrak{L}_{\mathrm{Hom} \circ \xi}(\varphi)\left(\nu^{1}, \ldots, \nu^{k}\right)=\mathfrak{L}_{T \circ \xi}\left(\varphi\left(\nu^{1}, \ldots, \nu^{k}\right)\right)-\sum_{i} \varphi\left(\nu^{1}, \ldots, \mathfrak{L}_{T^{i} \circ \xi} \nu^{i}, \ldots, \nu^{k}\right) .
$$

Then

$$
d^{T}\left(\varphi_{\star}\left(\Psi_{1}, \ldots, \Psi_{k}\right)\right)=\sum_{j=1}^{k}(-1)^{q_{1}+\cdots+q_{j-1}} \varphi_{\star}\left(\Psi_{1}, \ldots, d^{T^{j}} \Psi_{j}, \ldots, \Psi_{k}\right) .
$$

A form $\Psi \in \Omega_{A}(M ; \mathfrak{f})$ is called a horizontal form if $\iota_{\nu} \Psi=0$ for all $\nu \in \operatorname{Sec} \boldsymbol{g}$. The space of horizontal forms is denoted by $\Omega_{A, i}(M ; \mathfrak{f})$. According to $(1.2) \Omega_{A, i}(M ; \mathfrak{f})$ is a module over the algebra of real horizontal forms $\Omega_{A, i}(M) . \Omega_{A, i}(M)$ is stable under $d^{A}$.

2. Homomorphisms $\omega^{\wedge}$ and $\Omega^{\vee}$. Let $A=(A, \llbracket \cdot, \cdot \rrbracket, \gamma)$ be an arbitrary regular Lie algebroid over a foliated manifold $(M, E)$, and $\lambda: E \rightarrow A$ any connection in $A$, i.e. any splitting of its Atiyah sequence [K4]:

$$
0 \longrightarrow \boldsymbol{g} \longleftrightarrow A \frac{\gamma}{\longleftarrow \lambda} E \longrightarrow 0
$$

The linear homomorphism of graded vector spaces $\gamma_{\star}: \Omega_{E}(M ; \mathfrak{f}) \rightarrow \Omega_{A}(M ; \mathfrak{f})$ defined by the formula $\gamma_{\star}(\theta)\left(x ; \ldots, v_{i}, \ldots\right)=\theta\left(x ; \ldots, \gamma v_{i}, \ldots\right), v_{i} \in A_{\mid x}$, maps isomorphically $\Omega_{E}(M ; \mathfrak{f})$ onto the space of horizontal forms $\Omega_{A, i}(M ; \mathfrak{f})$. The inverse mapping is $\lambda_{\star}$ : $\Omega_{A, i}(M ; \mathfrak{f}) \rightarrow \Omega_{E}(M ; \mathfrak{f})$ defined by $\lambda_{\star}(\Psi)\left(x ; \ldots, w_{i}, \ldots\right)=\Psi\left(x ; \ldots, \lambda w_{i}, \ldots\right), w_{i} \in E_{\mid x}$. For the trivial vector bundle $f=M \times \mathbb{R}$, one can easily obtain the equality $d^{E}=\lambda_{\star} \circ d^{A} \circ \gamma_{\star}$ which is equivalent to

$$
\gamma_{\star} \circ d^{E}=d^{A} \circ \gamma_{\star}
$$

Let $\omega: A \rightarrow \boldsymbol{g}$ be the connection form of $\lambda$. The mapping $H=\mathrm{id}_{A}-\omega: A \rightarrow A$ is the horizontal projection of vectors from $A$. It determines the horizontal projection of forms $H_{\star}: \Omega_{A}(M ; \mathfrak{f}) \rightarrow \Omega_{A}(M ; \mathfrak{f})$ by $H_{\star}(\Psi)\left(x ; \ldots, v_{i}, \ldots\right)=\Psi\left(x ; \ldots, H v_{i}, \ldots\right)$. In [K4] the curvature tensor $\Omega_{b} \in \Omega_{E}^{2}(M ; \boldsymbol{g})$ of $\lambda$ is defined by $\Omega_{b}\left(X_{1}, X_{2}\right)=-\omega\left(\llbracket \lambda \circ X_{1}, \lambda \circ X_{2} \rrbracket\right)$, $X_{i} \in \operatorname{Sec} E$. We define the so-called curvature form of $\lambda$ as a horizontal 2-form $\Omega$ on the Lie algebroid $A$, with values in $\boldsymbol{g}$, by the formula

$$
\Omega\left(\xi_{1}, \xi_{2}\right)=-\omega\left(\llbracket H \circ \xi_{1}, H \circ \xi_{2} \rrbracket\right), \quad \xi_{i} \in \operatorname{Sec} A .
$$

Below, the exterior derivative of forms on the Lie algebroid $A$, with values in $\boldsymbol{g}$, [also in the associated vector bundles] with respect to the adjoint representation $\operatorname{ad}_{A}: A \rightarrow A(\boldsymbol{g})$ [or induced ones] will be briefly denoted by $d^{\boldsymbol{g}}$.

Proposition 2.1 (The Maurer-Cartan equation).

$$
\Omega=d^{g} \omega-\frac{1}{2}[\omega, \omega] .
$$

(The form $[\omega, \omega]$ is defined via (1.1) for the 2-linear homomorphism $[\cdot, \cdot]: \boldsymbol{g} \times \boldsymbol{g} \rightarrow \boldsymbol{g}$ where $[\cdot, \cdot]_{\mid x}: \boldsymbol{g}_{\mid x} \times \boldsymbol{g}_{\mid x} \rightarrow \boldsymbol{g}_{\mid x}$ is the Lie algebra structure of the isotropy Lie algebra $\left.\boldsymbol{g}_{\mid x}.\right)$

(Remark: The difference here, in comparison with the classical formula for principal bundles — the sign "-" before the second component — has its roots in the fact that the 
Lie algebra of the structure Lie group in the principal bundle considered there is taken left, not right.)

Pro of. Clearly, it is sufficient to notice that $\iota_{\nu} \Omega=0=\iota_{\nu}\left(d^{\boldsymbol{g}} \omega-\frac{1}{2}[\omega, \omega]\right), \nu \in \operatorname{Sec} \boldsymbol{g}$, and $H_{\star}(\Omega)=H_{\star}\left(d^{\boldsymbol{g}} \omega-\frac{1}{2}[\omega, \omega]\right)$.

2.1. Homomorphism. For each point $x \in M$, the mapping

$$
\rho: \boldsymbol{g}_{\mid x}^{\star} \longrightarrow A_{\mid x}^{\star}=\bigwedge^{1} A_{\mid x}^{\star} \subset \bigwedge A_{\mid x}^{\star}, w^{\star} \longmapsto w^{\star} \circ \omega_{\mid x},
$$

is linear and has the property $\rho\left(w^{\star}\right) \wedge \rho\left(w^{\star}\right)=0$ for $w^{\star} \in \boldsymbol{g}_{\mid x}^{\star} . \wedge A_{\mid x}^{\star}$ is an associative algebra with unit element, therefore, by the universal property of the exterior algebra $\bigwedge A_{\mid x}^{\star}$, see [G, p.103], we obtain the existence and uniqueness of a homomorphism of algebras of degree 0 ,

$$
\omega_{x}^{\wedge}: \wedge \boldsymbol{g}_{\mid x}^{\star} \longrightarrow \bigwedge A_{\mid x}^{\star}
$$

extending $\rho$ and such that $\omega_{x}^{\wedge}(1)=1$. Using the canonical duality between the exterior algebra over a vector space and over its dual [G, p.104] we have that

$$
\left\langle\omega_{x}^{\wedge}(\psi), w_{1} \wedge \ldots \wedge w_{k}\right\rangle=\left\langle\psi, \omega\left(x ; w_{1}\right) \wedge \ldots \wedge \omega\left(x ; w_{k}\right)\right\rangle
$$

for $\psi \in \bigwedge^{k} \boldsymbol{g}_{\mid x}^{\star}$ and $w_{i} \in A_{\mid x}$. We notice that if $\Psi \in \operatorname{Sec} \bigwedge^{k} \boldsymbol{g}^{\star}$, then

$$
\omega^{\wedge}(\Psi): M \longrightarrow \bigwedge^{k} A^{\star}, x \longmapsto \omega_{x}^{\wedge}(\Psi(x)),
$$

is a $C^{\infty}$ cross-section of $\bigwedge^{k} A^{\star}$, i.e. $\omega^{\wedge}(\Psi) \in \Omega_{A}^{k}(M)$. Of course

$$
\omega^{\wedge}: \bigoplus_{k \geq 0} \operatorname{Sec} \wedge^{k} g^{\star} \longrightarrow \Omega_{A}(M), \Psi \longmapsto \omega^{\wedge}(\Psi),
$$

is a homomorphism of algebras where the space $\bigoplus_{k>0} \operatorname{Sec} \bigwedge^{k} \boldsymbol{g}^{\star}$ is equipped with the structure $\left(\Psi_{1}, \Psi_{2}\right) \mapsto \Psi_{1} \wedge \Psi_{2}$ for which $\Psi_{1} \wedge \Psi_{2}$ is defined pointwise. Define a $C^{\infty}$ 2-linear homomorphism of vector bundles $\langle\cdot, \cdot\rangle: \Lambda^{k} \boldsymbol{g}^{\star} \times \Lambda^{k} \boldsymbol{g} \rightarrow \mathbb{R}$ via the family of the canonical dualities $\langle\cdot, \cdot\rangle_{x}: \bigwedge^{k} \boldsymbol{g}_{\mid x}^{\star} \times \bigwedge^{k} \boldsymbol{g}_{\mid x} \rightarrow \mathbb{R}$. Looking at formula (1.1) and treating $\Psi \in \operatorname{Sec} \Lambda^{k} \boldsymbol{g}^{\star}$ as a 0 -form on $A$, with values in $\Lambda^{k} \boldsymbol{g}^{\star}$, we can easily show

$$
\omega^{\wedge}(\Psi)=\frac{1}{k !}\langle\Psi, \underbrace{\omega \wedge \ldots \wedge \omega}_{k \text { times }}\rangle,
$$

where $\omega \wedge \ldots \wedge \omega$ is defined by formula (1.1) for the $k$-linear homomorphism

$$
\wedge: \boldsymbol{g} \times \ldots \times \boldsymbol{g} \longrightarrow \bigwedge^{k} \boldsymbol{g}
$$

whereas $\langle\Psi, \omega \wedge \ldots \wedge \omega\rangle$ - for the duality $\langle\cdot, \cdot\rangle$. In view of (1.2) and of (2.2) above, we have

$$
\iota_{\nu}\left(\omega^{\wedge}(\Psi)\right)=\omega^{\wedge}\left(\iota_{\nu}(\Psi)\right), \text { if } \nu \in \operatorname{Sec} \boldsymbol{g} .
$$

2.2. Homomorphism $\Omega^{\vee}$. Let $\Omega \in \Omega_{A}^{2}(M ; \boldsymbol{g})$ be the curvature form of the connection $\lambda$ under consideration. For each point $x \in M$, the mapping

$$
\mu: \boldsymbol{g}_{\mid x}^{\star} \longrightarrow \bigwedge^{2} A_{\mid x}^{\star} \subset \bigwedge^{\mathrm{ev}} A_{\mid x}^{\star}, w^{\star} \longmapsto w^{\star} \circ \Omega_{\mid x},
$$

is linear and has the property $\mu\left(u^{\star}\right) \wedge \mu\left(w^{\star}\right)=\mu\left(w^{\star}\right) \wedge \mu\left(u^{\star}\right)$ for $u^{\star}, w^{\star} \in \boldsymbol{g}_{\mid x}^{\star} \cdot \wedge^{\text {ev }} A_{\mid x}^{\star}$ is an associative algebra with unit element, therefore, by the universal symmetric algebra 
property of $\bigvee \boldsymbol{g}_{\mid x}^{\star}[\mathrm{G}, \mathrm{p} .192]$, there exists a unique homomorphism of algebras of degree 0

$$
\Omega_{x}^{\vee}: \bigvee g_{\mid x}^{\star} \longrightarrow \bigwedge^{\mathrm{ev}} A_{\mid x}^{\star}
$$

extending $\mu$ and such that $\Omega_{x}^{\vee}(1)=1$. Via the canonical dualities [G, pp.104, 193], the homomorphism $\Omega_{x}^{\vee}$ is defined by the formula

$$
\begin{aligned}
& \left\langle\Omega_{x}^{\vee}\left(\Gamma_{x}\right), w_{1} \wedge \ldots \wedge w_{2 k}\right\rangle \\
= & \frac{1}{k !} \cdot \frac{1}{2^{k}} \cdot \sum_{\sigma} \operatorname{sgn} \sigma \cdot\left\langle\Gamma, \Omega\left(x ; w_{\sigma(1)} \wedge w_{\sigma(2)}\right) \vee \ldots \vee \Omega\left(x ; w_{\sigma(2 k-1)} \wedge w_{\sigma(2 k)}\right)\right\rangle
\end{aligned}
$$

for $\Gamma_{x} \in \bigvee^{k} \boldsymbol{g}_{\mid x}^{\star}$ and $w_{i} \in A_{\mid x}$. Indeed, in view of the linearity with respect to $\Gamma_{x}$ of both sides of the above equality, it is sufficient to check it on the simple tensors $\Gamma_{x}=$ $w_{1}^{\star} \vee \ldots \vee w_{k}^{\star}, w_{i}^{\star} \in \boldsymbol{g}_{\mid x}^{\star}$.

$$
\begin{aligned}
& \left\langle\Omega_{x}^{\vee}\left(w_{1}^{\star} \vee \ldots \vee w_{k}^{\star}\right), w_{1} \wedge \ldots \wedge w_{2 k}\right\rangle \\
= & \left\langle\Omega_{x}^{\vee}\left(w_{1}^{\star}\right) \wedge \ldots \wedge \Omega_{x}^{\vee}\left(w_{k}^{\star}\right), w_{1} \wedge \ldots \wedge w_{2 k}\right\rangle \\
= & \frac{1}{2^{k}} \cdot \sum_{\sigma} \operatorname{sgn} \sigma \cdot w_{1}^{\star}\left(\Omega\left(x ; w_{\sigma(1)} \wedge w_{\sigma(2)}\right)\right) \cdot \ldots \cdot w_{k}^{\star}\left(\Omega\left(x ; w_{\sigma(2 k-1)} \wedge w_{\sigma(2 k)}\right)\right) \\
= & \frac{1}{k !} \cdot \frac{1}{2^{k}} \cdot \sum_{\sigma} \operatorname{sgn} \sigma \cdot \sum_{\tau} w_{1}^{\star}\left(\Omega\left(x ; w_{\sigma(2 \cdot \tau(1)-1)} \wedge w_{\sigma(2 \cdot \tau(1))}\right)\right) \cdot \ldots \\
& \ldots \cdot w_{k}^{\star}\left(\Omega\left(x ; w_{\sigma(2 \cdot \tau(k)-1)} \wedge w_{\sigma(2 \cdot \tau(k))}\right)\right) \\
= & \frac{1}{k !} \cdot \frac{1}{2^{k}} \cdot \sum_{\sigma} \operatorname{sgn} \sigma \cdot \operatorname{perm}\left[\left\langle w_{i}^{\star}, \Omega\left(x ; w_{\sigma(2 j-1)} \wedge w_{\sigma(2 j)}\right)\right\rangle ; i, j \leq k\right] \\
= & \frac{1}{k !} \cdot \frac{1}{2^{k}} \cdot \sum_{\sigma} \operatorname{sgn} \sigma \cdot\left\langle w_{1}^{\star} \vee \ldots \vee w_{k}^{\star}, \Omega\left(x ; w_{\sigma(1)} \wedge w_{\sigma(2)}\right) \vee \ldots\right. \\
& \left.\ldots \vee \Omega\left(x ; w_{\sigma(2 k-1)} \wedge w_{\sigma(2 k)}\right)\right\rangle .
\end{aligned}
$$

Applying (2.4), we see that, for $\Gamma \in \operatorname{Sec} \bigvee^{k} \boldsymbol{g}^{\star}$, the cross-section $\Omega^{\vee}(\Gamma)$ of $\bigwedge^{2 k} A^{\star}$ defined by $x \mapsto \Omega_{x}^{\vee}(\Gamma(x))$ is $C^{\infty}$, i.e. $\Omega^{\vee}(\Gamma) \in \Omega_{A}^{2 k}(M)$. The space $\bigoplus_{k \geq 0} \operatorname{Sec}^{k} \boldsymbol{g}^{\star}$ forms an algebra in a standard way, and the mapping

$$
\Omega^{\vee}: \bigoplus_{k \geq 0} \operatorname{Sec}^{k} \boldsymbol{g}^{\star} \longrightarrow \Omega_{A}^{\mathrm{ev}}(M), \quad \Gamma \longmapsto \Omega^{\vee}(\Gamma)
$$

is a homomorphism of algebras. By simple calculations, we obtain

$$
\Omega^{\vee}(\Gamma)=\frac{1}{k !} \cdot\langle\Gamma, \underbrace{\Omega \vee \ldots \vee \Omega}_{k \text { times }}\rangle \text { for } \Gamma \in \operatorname{Sec}^{k} \boldsymbol{g}^{\star}
$$

(the forms $\Omega \vee \ldots \vee \Omega$ and $\langle\Gamma, \Omega \vee \ldots \vee \Omega\rangle$ are defined by (1.1) for suitable multilinear homomorphisms).

It is well known that, in the vector space $\wedge \boldsymbol{g}_{\mid x}^{\star}$, the classical Chevalley-Eilenberg differential works, see, for example, [G-H-V, Vol.III, p.107]. For our purpose, we must slightly modify it by multiplying it by -1 (cf. Remark next to Prop.2.1), i.e. we adopt 
the following differential:

$$
\begin{aligned}
\delta_{x} & : \wedge \boldsymbol{g}_{\mid x}^{\star} \longrightarrow \wedge \boldsymbol{g}_{\mid x}^{\star} \\
\left\langle\delta_{x}(\psi), w_{0} \wedge \ldots \wedge w_{k}\right\rangle & =-\sum_{i<j}(-1)^{i+j}\left\langle\psi,\left[w_{i}, w_{j}\right] \wedge \ldots \hat{i} \ldots \hat{j} \ldots \wedge w_{k}\right\rangle
\end{aligned}
$$

for $\psi \in \Lambda^{k} \boldsymbol{g}_{\mid x}^{\star}(k \geq 1), w_{i} \in \boldsymbol{g}_{\mid x}$, and $\delta_{x} \psi=0$ for $\psi \in \Lambda^{0} \boldsymbol{g}_{\mid x}^{\star}$. $\delta_{x}$ is an antiderivation of degree +1 and, for an arbitrary $k \geq 0$, the induced homomorphism of vector bundles

$$
\delta^{k}: \bigwedge^{k} \boldsymbol{g}^{\star} \longrightarrow \bigwedge^{k+1} \boldsymbol{g}^{\star}
$$

is, obviously, $C^{\infty}$.

Applying the Maurer-Cartan equation, we get for $w^{\star} \in \operatorname{Sec} \boldsymbol{g}^{\star}$

$$
\Omega^{\vee}\left(w^{\star}\right)=\left\langle w^{\star}, d^{g} \omega\right\rangle-\omega^{\wedge}\left(\delta\left(w^{\star}\right)\right) .
$$

Define the mapping

$$
K: \bigoplus_{k \geq 0} \operatorname{Sec} \bigwedge^{k} \boldsymbol{g}^{\star} \longrightarrow \Omega_{A}(M)
$$

by the formula

$$
K(\Psi)=\frac{1}{k !} \cdot\left\langle\Psi, d^{\boldsymbol{g}}(\underbrace{\omega \wedge \ldots \wedge \omega}_{k \text { times }})\right\rangle-\omega^{\wedge}(\delta \Psi) \text { for } \Psi \in \operatorname{Sec} \bigwedge^{k} \boldsymbol{g}^{\star} .
$$

Of course, by (2.6),

$$
K\left(w^{\star}\right)=\Omega^{\vee}\left(w^{\star}\right) \text { if } w^{\star} \in \operatorname{Sec} \boldsymbol{g}^{\star} .
$$

Proposition 2.2. The fundamental formulae for $K$ :

(1) $K\left(w_{1}^{\star} \wedge \ldots \wedge w_{k}^{\star}\right)=\sum_{s=1}^{k}(-1)^{s-1} K\left(w_{s}^{\star}\right) \wedge \omega^{\wedge}\left(w_{1}^{\star} \wedge \ldots \hat{s} \ldots \wedge w_{k}^{\star}\right)$ for $w_{s}^{\star} \in \operatorname{Sec} \boldsymbol{g}^{\star}$

(2) $K(\Psi)=d^{A}\left(\omega^{\wedge}(\Psi)\right)-\omega^{\wedge}(\delta \Psi)-\frac{1}{k !} \cdot\langle d^{\boldsymbol{g}} \Psi, \underbrace{\omega \wedge \ldots \wedge \omega}_{k \text { times }}\rangle \quad$ for $\Psi \in \operatorname{Sec} \wedge^{k} \boldsymbol{g}^{\star}$.

Pr o of. (1): Applying (1.3), we get

$$
\begin{aligned}
& K\left(w_{1}^{\star} \wedge \ldots \wedge w_{k}^{\star}\right) \\
= & \frac{1}{(k-1) !} \cdot\left\langle w_{1}^{\star} \wedge \ldots \wedge w_{k}^{\star},\left(d^{\boldsymbol{g}} \omega\right) \wedge \omega \wedge \ldots \wedge \omega\right\rangle \\
& -\sum_{s=1}^{k}(-1)^{s-1} \omega^{\wedge}\left(\delta w_{s}^{\star}\right) \wedge \omega^{\wedge}\left(w_{1}^{\star} \wedge \ldots \wedge \hat{s} \ldots \wedge w_{k}^{\star}\right) .
\end{aligned}
$$

On the other hand,

$$
\begin{aligned}
& \sum_{s=1}^{k}(-1)^{s-1} K\left(w_{s}^{\star}\right) \wedge \omega^{\wedge}\left(w_{1}^{\star} \wedge \ldots \hat{s} \ldots \wedge w_{k}^{\star}\right) \\
= & \sum_{s=1}^{k}(-1)^{s-1}\left(\left\langle w_{s}^{\star}, d^{\boldsymbol{g}} \omega\right\rangle-\omega^{\wedge}\left(\delta w_{s}^{\star}\right)\right) \wedge \omega^{\wedge}\left(w_{1}^{\star} \wedge \ldots \hat{s} \ldots \wedge w_{k}^{\star}\right),
\end{aligned}
$$


therefore, it is sufficient to check the equality

$$
\begin{aligned}
& \frac{1}{(k-1) !} \cdot\left\langle w_{1}^{\star} \wedge \ldots \wedge w_{k}^{\star},\left(d^{\boldsymbol{g}} \omega\right) \wedge \omega \wedge \ldots \wedge \omega\right\rangle \\
= & \sum_{s=1}^{k}(-1)^{s-1}\left\langle w_{s}^{\star}, d^{\boldsymbol{g}} \omega\right\rangle \wedge \omega^{\wedge}\left(w_{1}^{\star} \wedge \ldots \hat{s} \ldots \wedge w_{k}^{\star}\right) .
\end{aligned}
$$

(2): By (1.3) (treating $\Psi$ as a 0 -form on the Lie algebroid $A$, with values in $\bigwedge^{k} \boldsymbol{g}^{\star}$ ), we have $d^{A}\langle\Psi, \omega \wedge \ldots \wedge \omega\rangle=\left\langle d^{\boldsymbol{g}} \Psi, \omega \wedge \ldots \wedge \omega\right\rangle+\left\langle\Psi, d^{\boldsymbol{g}}(\omega \wedge \ldots \wedge \omega)\right\rangle$. Therefore, by (2.2), $K(\Psi)=d^{A}\left(\omega^{\wedge}(\Psi)\right)-\omega^{\wedge}(\delta \Psi)-\frac{1}{k !} \cdot\left\langle d^{\boldsymbol{g}} \Psi, \omega \wedge \ldots \wedge \omega\right\rangle$.

Since each cross-section $\Psi \in \operatorname{Sec} \bigwedge^{k} \boldsymbol{g}^{\star}$ is locally a sum of cross-sections of the form $w_{1}^{\star} \wedge \ldots \wedge w_{k}^{\star}$ for $w_{s}^{\star} \in \operatorname{Sec} \boldsymbol{g}^{\star}$, we get

Corollary 2.3. If the connection $\lambda$ considered is flat (i.e. $\Omega=0$ ), then, according to (2.8) and Prop. $2.2(1)$, we see that $K \equiv 0$, which means, by (2.7) and Prop. 2.2(2), that

$$
\begin{aligned}
\omega^{\wedge}(\delta \Psi) & =\frac{1}{k !} \cdot\left\langle\Psi, d^{g}(\omega \wedge \ldots \wedge \omega)\right\rangle \\
& =d^{A}\left(\omega^{\wedge}(\Psi)\right)-\frac{1}{k !} \cdot\left\langle d^{\boldsymbol{g}} \Psi, \omega \wedge \ldots \wedge \omega\right\rangle .
\end{aligned}
$$

REMARK 1. Assume $\Omega=0$. If $\Psi \in \operatorname{Sec} \bigwedge^{k} \boldsymbol{g}^{\star}$ is invariant with respect to the representation $\operatorname{ad}_{A}$ of $A$ on $\boldsymbol{g}$ (equivalently, if $\left.d^{\boldsymbol{g}} \Psi=0\right)$, then $d^{A}\left(\omega^{\wedge} \Psi\right)=0$. Indeed, by Cor.2.3, we have $d^{A}\left(\omega^{\wedge} \Psi\right)=\omega^{\wedge}(\delta \Psi)$; but, for each point $x \in M$, the tensor $\Psi(x) \in \wedge^{k} \boldsymbol{g}_{\mid x}^{\star}$ is invariant under the canonical representation of the Lie algebra $\boldsymbol{g}_{\mid x}$ on $\bigwedge^{k} \boldsymbol{g}_{\mid x}^{\star}$ and such a tensor is a cycle [G-H-V, Vol.III, p.186], so $(\delta \Psi)(x)=\delta_{x}\left(\Psi_{x}\right)=0$. Therefore, there exists a homomorphism of algebras

$$
\omega^{\#}: \bigoplus_{k \geq 0}\left(\operatorname{Sec} \bigwedge^{k} \boldsymbol{g}^{\star}\right)_{I^{0}} \longrightarrow Z_{A}(M) \longrightarrow H_{A}(M), \quad \Psi \longmapsto\left[\omega^{\wedge}(\Psi)\right] .
$$

3. A construction of the characteristic classes of flat regular Lie algebroids. Here we construct characteristic classes having the following property:

- the existence of nontrivial classes among them is a measure of the incompatibility of the flat structure of a given regular Lie algebroid $A($ over $(M, E))$ with a given subalgebroid $B$ of $A$ (also over $(M, E)$ ).

In the case of an integrable transitive Lie algebroid $A=A(P), P$ being any principal bundle, these classes agree with the so-called characteristic classes of the flat principal bundle $P[\mathrm{~K}-\mathrm{T}]$.

Consider in a given regular Lie algebroid $\left(A, \llbracket \cdot, \cdot \rrbracket, \gamma_{A}\right)$ over $(M, E)$ two geometric structures:

(1) a flat connection $\lambda: E \rightarrow A$,

(2) a subalgebroid $B \subset A$ over $(M, E)$, as in the following diagram: 


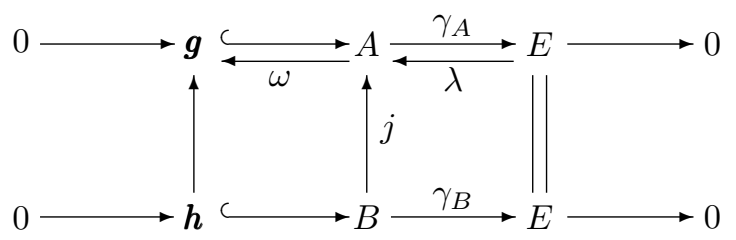

Notice that $\boldsymbol{h}=\boldsymbol{g} \cap B\left(\boldsymbol{h}:=\operatorname{ker} \gamma_{B}\right)$. The system $(A, \lambda, B)$ will then be called an FS-regular Lie algebroid (over $(M, E))$.

Example 3.1. (1) A triad $\left(P, P^{\prime}, \omega\right)$ consisting of a principal bundle $P$, of an $H$ reduction $P^{\prime}$ and a flat connection in $P$ with connection form $\omega$ determines an FStransitive Lie algebroid $\left(A(P), \lambda, A\left(P^{\prime}\right)\right)$ ( $\lambda$ corresponds to $\omega$ ). For the theory of flat principal bundles with given reductions, see $[\mathrm{K}-\mathrm{T}]$.

(2) We recall that a transitive Lie algebroid $A=\left(A, \llbracket \cdot, \cdot \rrbracket, \gamma_{A}\right)$ on $M$ together with an involutive distribution $F \subset T M$ give rise to the regular Lie algebroid over $(M, F)$ of the form $A^{F}=\gamma_{A}^{-1}[F] \subset A$, see [K4, s.1.1.3]. Consider now a triple $(A, B, \lambda)$ consisting of a transitive Lie algebroid $A$ on $M$, a transitive Lie subalgebroid $B$ of $A$ and a partially flat connection $\lambda$ in $A$, over a given involutive distribution $F \subset T M$. The triple

$$
\left(A^{F}, B^{F}, \lambda \mid F\right)
$$

is an FS-regular Lie algebroid over $(M, F)$.

(3) Let now the system $\left(P, P^{\prime}, \omega\right)$ be given as in example (1) above with the difference that $\omega$ is assumed to be partially flat, say, over an involutive distribution $F \subset T M$. Such a system (named a foliated bundle) is investigated, for example, in [K-T]. It determines the (nontransitive) FS-regular Lie algebroid $\left(A(P)^{F}, A\left(P^{\prime}\right)^{F}, \lambda \mid F\right)$, as above.

(4) Let $(M, F)$ be any transversally complete foliation with the basic fibration $\pi_{b}$ : $M \rightarrow W$. Denote by $A(M, F)$ its transitive Lie algebroid on $W$ defined by P. Molino in 1977 [MO1]. Let $F_{b} \subset T M$ be the vector bundle tangent to the basic foliation. Denote, as usual, by $L(M, F)$ the space of foliate vector fields and by $\alpha: T M \rightarrow Q=T M / F$, $\beta: Q \rightarrow A(M, F)$ the canonical linear homomorphisms (see [K4]). It is easy to see that:

- There is a 1-1 correspondence between transitive Lie subalgebroids

$$
B \subset A(M, F)
$$

and involutive vector subbundles $\tilde{B} \subset T M$ such that

(1) $F \subset \tilde{B}$,

(2) $F_{b}+\tilde{B}=T M$,

(3) the Lie algebra $\operatorname{Sec}(\tilde{B}) \cap L(M, F)$ generates at each point $x \in M$ the entire space $\tilde{B}_{\mid x}$.

The correspondence $B \mapsto \tilde{B}$ is established in such a way that

$$
\tilde{B}_{\mid x}=\alpha_{x}^{-1}\left[\beta_{x}^{-1}\left[B_{\mid \pi_{b}(x)}\right]\right] .
$$

- There is a 1-1 correspondence between connections $\lambda$ in $A(M, F)$ and distributions $C \subset T M$ such that

(a) $F_{b} \cap C=F$, 
(b) $F_{b}+C=T M$,

(c) $\operatorname{Sec} C \cap L(M, F)$ generates at each point $x \in M$ the entire vector space $C_{\mid x}$.

(The correspondence: $\lambda \mapsto C:=\alpha^{-1}\left[\beta^{-1}[\operatorname{Im} \lambda]\right]$.) Such a distribution $C$ exists (because every Lie algebroid has a connection) and $C$ is involutive if and only if the corresponding connection $\lambda$ is flat. Each distribution $C$ on $M$ satisfying (a) $\div$ (c) above is called an F-connection.

For the Lie algebroid $A(G ; H)$ of the TC-foliation of left cosets of a nonclosed Lie subgroup $H$ in a connected Lie group $G$, conditions (3) and (c) above are equivalent to (3') and (c') below, respectively:

$\left(3^{\prime}\right)$, (c') $\tilde{B}$ (and $C$ ) is $C^{\infty}$ and $\bar{H}$-right-invariant.

Thus, for a given TC-foliation $(M, F)$, the triad $(A(M, F), C, \tilde{B})$ where $C \subset T M$ is an involutive distribution fulfilling (a), (b), (c) above, and $\tilde{B} \subset T M$ is an involutive distribution fulfilling (1), (2), (3) above, yields an FS-transitive Lie algebroid. Using the restriction $A(M, F)^{E}$ (to a foliation $E$ on the basic manifold $W$ ), we can obtain an FS-regular Lie algebroid over $(W, E)$.

We construct some characteristic classes of an FS-regular Lie algebroid $(A, \lambda, B)$, measuring the independence of $\lambda$ and $B$, i.e. to what extent $\operatorname{Im} \lambda$ is not contained in $B$. The construction has a number of steps.

Let $\boldsymbol{s}: \boldsymbol{g} \rightarrow \boldsymbol{g} / \boldsymbol{h}$ be the canonical projection. Applying (2.3), we can easily obtain that the form $\varphi(\Psi):=\omega^{\wedge}\left(\bigwedge^{k} \boldsymbol{s}^{\star} \circ \Psi\right), \Psi \in \operatorname{Sec} \bigwedge^{k}(\boldsymbol{g} / \boldsymbol{h})^{\star}$, is $\boldsymbol{h}$-horizontal, i.e., equivalently, its restriction to the subalgebroid $B, j^{\star}\left(\omega^{\wedge}\left(\bigwedge^{k} s^{\star} \circ \Psi\right)\right)$, is horizontal. Therefore there exists a form $\Delta \Psi \in \Omega_{E}^{k}(M)$ such that

$$
j^{\star}\left(\omega^{\wedge}\left(\bigwedge^{k} s^{\star} \circ \Psi\right)\right)=\left(\gamma_{B}\right)_{\star}(\Delta \Psi) .
$$

Notice that if $\lambda$ is a connection in $B$ (i.e. $\operatorname{Im} \lambda \subset B$ ), then $\Delta \Psi=0$.

Put $\Delta: \bigoplus_{k \geq 0} \operatorname{Sec} \bigwedge^{k}(\boldsymbol{g} / \boldsymbol{h})^{\star} \rightarrow \Omega_{E}(M), \Psi \mapsto \Delta \Psi$. The mapping $\Delta$ is a superposition of homomorphisms of algebras:

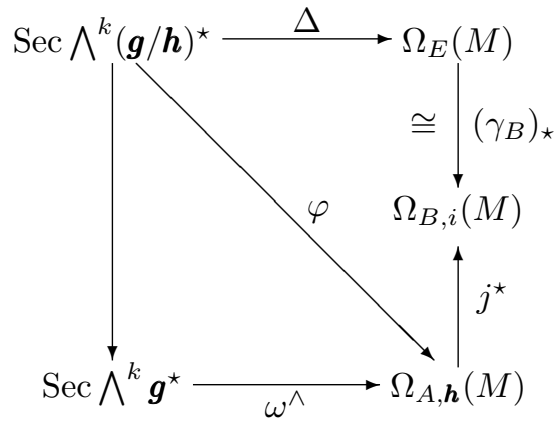

(here $\Omega_{A, \boldsymbol{h}}(M)$ denotes the space of $\boldsymbol{h}$-horizontal forms on $A$ ). Hence it is itself such a homomorphism.

Directly, $\Delta$ is defined by the formula

$$
(\Delta \Psi)\left(x ; w_{1} \wedge \ldots \wedge w_{k}\right)=\left\langle\Psi_{x} ;\left[\omega\left(x ; \tilde{w}_{1}\right)\right] \wedge \ldots \wedge\left[\omega\left(x ; \tilde{w}_{k}\right)\right]\right\rangle
$$

for $\tilde{w}_{i} \in B_{\mid x}$ such that $\gamma_{B}\left(\tilde{w}_{i}\right)=w_{i}, w_{i} \in E_{\mid x}, x \in M$. 
Define a representation $\operatorname{ad}_{B, \boldsymbol{g}}^{\wedge}: B \rightarrow A\left(\bigwedge^{k}(\boldsymbol{g} / \boldsymbol{h})^{\star}\right)$ by the formula

$$
\begin{aligned}
& \left\langle\mathfrak{L}_{\mathrm{ad}_{B, \boldsymbol{g}} \circ \xi}(\Psi),\left[\nu_{1}\right] \wedge \ldots \wedge\left[\nu_{k}\right]\right\rangle \\
= & \left(\gamma_{B} \circ \xi\right)\left\langle\Psi,\left[\nu_{1}\right] \wedge \ldots \wedge\left[\nu_{k}\right]\right\rangle-\sum_{j=1}^{k}\left\langle\Psi,\left[\nu_{1}\right] \wedge \ldots \wedge\left[\llbracket \xi, \nu_{j} \rrbracket\right] \wedge \ldots \wedge\left[\nu_{k}\right]\right\rangle
\end{aligned}
$$

for $\Psi \in \operatorname{Sec} \bigwedge^{k}(\boldsymbol{g} / \boldsymbol{h})^{\star}, \xi \in \operatorname{Sec} B$, and $\nu_{j} \in \operatorname{Sec} \boldsymbol{g}$. The correctness of this definition follows from the fact that if one of $\nu_{j}$ 's lies in $\boldsymbol{h}$, then $\llbracket \xi, \nu_{j} \rrbracket$ lies in $\boldsymbol{h}$, too. Notice that $\operatorname{ad}_{B, \boldsymbol{g}}^{\wedge}=\bigwedge^{k}\left(\operatorname{ad}_{B, \boldsymbol{g}}\right)^{\natural}[\mathrm{K} 4,2.1 .2-3]$ where $\operatorname{ad}_{B, \boldsymbol{g}}: B \rightarrow A(\boldsymbol{g} / \boldsymbol{h})$ is a representation given by

$$
\mathfrak{L}_{\mathrm{ad}_{B, \boldsymbol{g}} \circ \xi}([\nu])=\left[\mathfrak{L}_{\mathrm{ad}_{A} \circ \xi}(\nu)\right]=[\llbracket \xi, \nu \rrbracket]
$$

for $\xi \in \operatorname{Sec} B$ and $\nu \in \operatorname{Sec} \boldsymbol{g}$.

In the space $\bigoplus_{k \geq 0}\left(\operatorname{Sec} \bigwedge^{k}(\boldsymbol{g} / \boldsymbol{h})^{\star}\right)_{I^{0}}$ of cross-sections invariant with respect to ad $\wedge_{B, \boldsymbol{g}}^{\wedge}$, we introduce a differential $\bar{\delta}$ of degree +1 defined as follows: for $\Psi \in\left(\operatorname{Sec} \bigwedge^{k}(\boldsymbol{g} / \boldsymbol{h})^{\star}\right)_{I^{0}}$ and $\nu_{j} \in \operatorname{Sec} \boldsymbol{g}$, we put

$$
\left\langle\bar{\delta} \Psi,\left[\nu_{0}\right] \wedge \ldots \wedge\left[\nu_{k}\right]\right\rangle=-\sum_{i<j}(-1)^{i+j}\left\langle\Psi,\left[\llbracket \nu_{i}, \nu_{j} \rrbracket\right] \wedge\left[\nu_{0}\right] \wedge \ldots \hat{i} \ldots \hat{j} \ldots \wedge\left[\nu_{k}\right]\right\rangle .
$$

The correctness of this definition is obvious (by the invariance of $\Psi$ and the equality $\left.\gamma_{B} \circ \nu_{j_{0}}=0\right)$.

To see the invariance of $\bar{\delta} \Psi$, take $\xi \in \operatorname{Sec} B$ and $\nu_{j} \in \operatorname{Sec} \boldsymbol{g}$. From the invariance of $\Psi$ we get

$$
\begin{aligned}
& \left(\gamma_{B} \circ \xi\right)\left\langle\bar{\delta} \Psi,\left[\nu_{0}\right] \wedge \ldots \wedge\left[\nu_{k}\right]\right\rangle \\
= & -\sum_{i<j}(-1)^{i+j}\left(\left\langle\Psi,\left[\llbracket \xi, \llbracket \nu_{i}, \nu_{j} \rrbracket \rrbracket\right] \wedge\left[\nu_{0}\right] \wedge \ldots \hat{i} \ldots \hat{j} \ldots \wedge\left[\nu_{k}\right]\right\rangle+\right. \\
& \left.+\sum_{s, s \notin\{i, j\}}\left\langle\Psi,\left[\llbracket \nu_{i}, \nu_{j} \rrbracket\right] \wedge\left[\nu_{0}\right] \wedge \ldots \wedge\left[\llbracket \xi, \nu_{s} \rrbracket\right] \wedge \ldots \hat{i} \ldots \hat{j} \ldots \wedge\left[\nu_{k}\right]\right\rangle\right) \\
= & -\sum_{s}\left(\sum_{s \neq i<j \neq s}(-1)^{i+j}\left\langle\Psi,\left[\llbracket \nu_{i}, \nu_{j} \rrbracket\right] \wedge\left[\nu_{0}\right] \wedge \ldots \wedge\left[\llbracket \xi, \nu_{s} \rrbracket\right] \wedge \ldots \hat{i} \ldots \hat{j} \ldots \wedge\left[\nu_{k}\right]\right\rangle\right. \\
& +\sum_{i<s}(-1)^{i+s}\left\langle\Psi,\left[\llbracket \nu_{i}, \llbracket \xi, \nu_{s} \rrbracket \rrbracket\right] \wedge\left[\nu_{0}\right] \wedge \ldots \hat{i} \ldots \hat{j} \ldots \wedge\left[\nu_{k}\right]\right\rangle+ \\
& \left.+\sum_{s<j}(-1)^{s+j}\left\langle\Psi,\left[\llbracket \xi, \llbracket \nu_{s}, \nu_{j} \rrbracket \rrbracket\right] \wedge\left[\nu_{0}\right] \wedge \ldots \hat{i} \ldots \hat{j} \ldots \wedge\left[\nu_{k}\right]\right\rangle\right) \\
= & \sum_{s}\left\langle\bar{\delta} \Psi,\left[\nu_{0}\right] \wedge \ldots \wedge\left[\llbracket \xi, \nu_{s} \rrbracket\right] \wedge \ldots \wedge\left[\nu_{k}\right]\right\rangle .
\end{aligned}
$$

It remains to notice that

(i) $\bar{\delta}^{2}=0$,

(ii) $\bar{\delta}$ is an antiderivation of degree +1 .

For this purpose, firstly, for an arbitrary point $x \in M$, we can define a space of tensors $\left(\bigwedge^{k}\left(\boldsymbol{g}_{\mid x} / \boldsymbol{h}_{\mid x}\right)^{\star}\right)_{I^{0}}$ invariant with respect to the representation of the Lie algebra 
$\boldsymbol{h}_{\mid x}$, induced on $\bigwedge^{k}\left(\boldsymbol{g}_{\mid x} / \boldsymbol{h}_{\mid x}\right)^{\star}$ by the representation ad $\mathrm{d}_{x}^{\star}$ of $\boldsymbol{h}_{\mid x}$ on $\left(\boldsymbol{g}_{\mid x} / \boldsymbol{h}_{\mid x}\right)^{\star}$ defined as follows: $\left\langle\operatorname{ad}_{x}^{\star}(\nu)(\psi),[\mu]\right\rangle=-\langle\psi,[[\nu, \mu]]\rangle$ for $\nu \in \boldsymbol{h}_{\mid x}, \psi \in\left(\boldsymbol{g}_{\mid x} / \boldsymbol{h}_{\mid x}\right)^{\star}$ and $\mu \in \boldsymbol{g}_{\mid x}$. Secondly, we define an antiderivation

$$
\bar{\delta}_{x}:\left(\bigwedge^{k}\left(\boldsymbol{g}_{\mid x} / \boldsymbol{h}_{\mid x}\right)^{\star}\right)_{I^{0}} \longrightarrow\left(\bigwedge^{k}\left(\boldsymbol{g}_{\mid x} / \boldsymbol{h}_{\mid x}\right)^{\star}\right)_{I^{0}}
$$

of degree +1 as the one which on elements $\psi$ of degree +1 equals $\left\langle\bar{\delta}_{x}(\psi),[\nu] \wedge[\mu]\right\rangle=$ $\langle\psi,[[\nu, \mu]]\rangle, \nu, \mu \in \boldsymbol{g}_{\mid x}$. It can easily be seen that if $\Psi \in\left(\operatorname{Sec} \bigwedge^{k}(\boldsymbol{g} / \boldsymbol{h})^{\star}\right)_{I^{0}}$, then $\Psi_{x} \in$ $\left(\bigwedge^{k}\left(\boldsymbol{g}_{\mid x} / \boldsymbol{h}_{\mid x}\right)^{\star}\right)_{I^{0}}$, and $(\bar{\delta} \Psi)_{x}=\bar{\delta}_{x}\left(\Psi_{x}\right)$.

In consequence, $\bar{\delta}$ fulfils (i) and (ii) in an evident manner. Of course, these properties of $\bar{\delta}$ can also be checked directly.

Definition 3.2. The relative cohomology algebra of $\boldsymbol{g}$ with respect to $B$ is defined as the cohomology algebra of the complex $\left(\bigoplus_{k \geq 0}\left(\operatorname{Sec} \bigwedge^{k}(\boldsymbol{g} / \boldsymbol{h})^{\star}\right)_{I^{0}}, \bar{\delta}\right)$

$$
H(\boldsymbol{g}: B):=H^{\star}\left(\bigoplus_{k \geq 0}\left(\operatorname{Sec} \bigwedge^{k}(\boldsymbol{g} / \boldsymbol{h})^{\star}\right)_{I^{0}}, \bar{\delta}\right) .
$$

Proposition 3.3. The mapping $\Delta$ restricted to the invariant cross-sections

$$
\Delta_{\star}=\Delta_{(A, \lambda, B)}: \bigoplus_{k \geq 0}\left(\operatorname{Sec} \bigwedge^{k}(\boldsymbol{g} / \boldsymbol{h})^{\star}\right)_{I^{0}} \longrightarrow \Omega_{E}(M), \quad \Psi \longmapsto \Delta \Psi,
$$

commutes with the differentials $\bar{\delta}$ and $d^{E}$.

Proof. We need to prove the equality

$$
\Delta(\bar{\delta} \Psi)=d^{E}(\Delta \Psi)
$$

for invariant cross-sections $\Psi$. The fact that $\left(\gamma_{B}\right)_{\star}$ is a monomorphism implies that this equality is equivalent to $\left(\gamma_{B}\right)_{\star}(\Delta(\bar{\delta} \Psi))=\left(\gamma_{B}\right)_{\star}\left(d^{E}(\Delta \Psi)\right)$. But, by definition $\left(\gamma_{B}\right)_{\star}(\Delta(\bar{\delta} \Psi))=j^{\star}\left(\omega^{\wedge}\left(\bigwedge^{k+1} \boldsymbol{s}^{\star} \circ(\bar{\delta} \Psi)\right)\right)$. On the other hand, applying (2.1) and the obvious fact $d^{B}\left(j^{\star} \Psi\right)=j^{\star}\left(d^{A} \Psi\right)$, we get

$$
\left(\gamma_{B}\right)_{\star}\left(d^{E}(\Delta \Psi)\right)=d^{B}\left(\left(\gamma_{B}\right)_{\star}(\Delta \Psi)\right)=j^{\star}\left(d^{A}\left(\omega^{\wedge}\left(\bigwedge^{k} \boldsymbol{s}^{\star} \circ \Psi\right)\right)\right) .
$$

Therefore, to prove (3.2), it remains to check that the forms $\omega^{\wedge}\left(\bigwedge^{k+1} \boldsymbol{s}^{\star} \circ(\bar{\delta} \Psi)\right)$ and $d^{A}\left(\omega^{\wedge}\left(\bigwedge^{k} \boldsymbol{s}^{\star} \circ \Psi\right)\right)$ agree on the cross-sections of $B$. Let $\xi_{0}, \ldots, \xi_{k} \in \operatorname{Sec} B$; then (see $(2.5))$

$$
\begin{aligned}
& \left\langle\omega^{\wedge}\left(\bigwedge^{k+1} \boldsymbol{s}^{\star} \circ(\bar{\delta} \Psi)\right), \xi_{0} \wedge \ldots \wedge \xi_{k}\right\rangle \\
= & -\sum_{i<j}(-1)^{i+j}\left\langle\Psi,\left[\llbracket \omega\left(\xi_{i}\right), \omega\left(\xi_{j}\right) \rrbracket\right] \wedge \ldots \hat{i} \ldots \hat{j} \ldots\right\rangle \\
= & -\sum_{i<j}(-1)^{i+j}\left\langle\bigwedge^{k} \boldsymbol{s}^{\star} \circ \Psi, \llbracket \omega\left(\xi_{i}\right), \omega\left(\xi_{j}\right) \rrbracket \wedge \ldots \hat{i} \ldots \hat{j} \ldots\right\rangle \\
= & \left\langle\omega^{\wedge}\left(\delta \circ \bigwedge^{k} \boldsymbol{s}^{\star} \circ \Psi\right), \xi_{0} \wedge \ldots \wedge \xi_{k}\right\rangle .
\end{aligned}
$$

On the other hand, by Prop.2.2(2) and the flatness of $\lambda$, we have

$$
d^{A}\left(\omega^{\wedge}\left(\bigwedge^{k} \boldsymbol{s}^{\star} \circ \Psi\right)\right)=\omega^{\wedge}\left(\delta \circ \bigwedge^{k} \boldsymbol{s}^{\star} \circ \Psi\right)+\frac{1}{k !} \cdot\langle d^{\boldsymbol{g}}\left(\bigwedge^{k} \boldsymbol{s}^{\star} \circ \Psi\right), \underbrace{\omega \wedge \ldots \wedge \omega}_{k \text { times }}\rangle .
$$

So, it remains to notice that $j^{\star}\left\langle d^{g}\left(\bigwedge^{k} \boldsymbol{s}^{\star} \circ \Psi\right), \omega \wedge \ldots \wedge \omega\right\rangle=0$. 
The above Proposition yields as a corollary

THEOREM 3.4. The mapping

$$
\Delta_{\#}: H(\boldsymbol{g}, B) \longrightarrow H_{E}(M),[\Psi] \longmapsto\left[\Delta_{\star} \Psi\right],
$$

is a correctly defined homomorphism of algebras.

The mapping $\Delta_{\#}$ is called the characteristic homomorphism of the FS-regular Lie algebroid $(A, \lambda, B)$. Its image $\operatorname{Im} \Delta_{\#} \subset H_{E}(M)$ is a subalgebra of $H_{E}(M)$, called the characteristic algebra of the FS-regular Lie algebroid $(A, \lambda, B)$, and its elements are the characteristic classes of that algebroid.

The compatibility of $\lambda$ with $B$ implies the vanishing of $\Delta_{\#}$ [of course, already on the level of forms]. $\Delta_{\#}$ is then a measure of the incompatibility of $\lambda$ with $B$.

\section{Functoriality}

Definition 4.1. Let $\left(A^{\prime}, \lambda^{\prime}, B^{\prime}\right)$ and $(A, \lambda, B)$ be two FS-regular Lie algebroids over $\left(M^{\prime}, E^{\prime}\right)$ and $(M, E)$, respectively. By a homomorphism

$$
H:\left(A^{\prime}, \lambda^{\prime}, B^{\prime}\right) \longrightarrow(A, \lambda, B)
$$

between them we mean a homomorphism $H: A^{\prime} \rightarrow A$ of regular Lie algebroids, say over $f:\left(M^{\prime}, E^{\prime}\right) \rightarrow(M, E)$, such that

(1) $H \circ \lambda^{\prime}=\lambda \circ f_{\star}$,

(2) $H\left[B^{\prime}\right] \subset B$.

Notice that $H^{\prime}=H \mid B^{\prime}: B^{\prime} \rightarrow B$ is then a homomorphism of regular Lie algebroids, too:

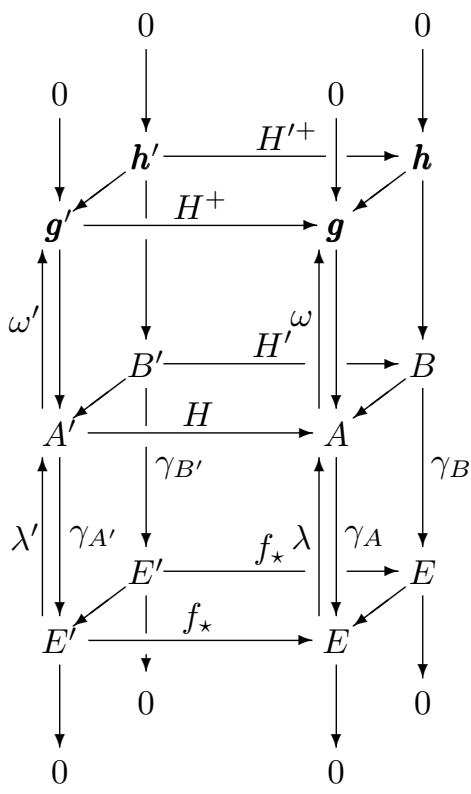


By the pullback of an FS-regular Lie algebroid $(A, \lambda, B)$ over $(M, E)$ via a mapping $f:\left(M^{\prime}, E^{\prime}\right) \rightarrow(M, E)$ we mean the FS-regular Lie algebroid $\left(f^{\wedge} A, \bar{\lambda}, f^{\wedge} B\right)$ where $\bar{\lambda}$ is the pullback of the connection $\lambda$, see definition 3.2.1 from [K4].

Notice that $\operatorname{pr}_{2}: f^{\wedge} A=E^{\prime} \times_{\left(f_{\star}, \gamma\right)} A \rightarrow A$ is a homomorphism of FS-regular Lie algebroids, called canonical. In view of the equality $\bar{H} \circ \lambda^{\prime}=\bar{\lambda}$, any homomorphism $H:\left(A^{\prime}, \lambda^{\prime}, B^{\prime}\right) \rightarrow(A, \lambda, B)$ of FS-regular Lie algebroids can be represented in the form of a superposition of a strong homomorphism with the canonical one:

$$
\left(A^{\prime}, \lambda^{\prime}, B^{\prime}\right) \stackrel{\bar{H}}{\longrightarrow}\left(f^{\wedge} A, \bar{\lambda}, f^{\wedge} B\right) \stackrel{\mathrm{pr}_{2}}{\longrightarrow}(A, \lambda, B) .
$$

Let $H:\left(A^{\prime}, \lambda^{\prime}, B^{\prime}\right) \rightarrow(A, \lambda, B)$ be a homomorphism of FS-regular Lie algebroids, see diagram above. We define the pullback

$$
H^{+\star}: \operatorname{Sec} \bigwedge^{k}(\boldsymbol{g} / \boldsymbol{h})^{\star} \longrightarrow \operatorname{Sec} \bigwedge^{k}\left(\boldsymbol{g}^{\prime} / \boldsymbol{h}^{\prime}\right)^{\star}
$$

by the formula

$$
\left\langle H^{+\star}(\Psi)_{x},\left[w_{1}^{\prime}\right] \wedge \ldots \wedge\left[w_{k}^{\prime}\right]\right\rangle=\left\langle\Psi_{f(x)},\left[H^{+}\left(w_{1}^{\prime}\right)\right] \wedge \ldots \wedge\left[H^{+}\left(w_{k}^{\prime}\right)\right]\right\rangle
$$

where $\Psi \in \operatorname{Sec} \bigwedge^{k}(\boldsymbol{g} / \boldsymbol{h})^{\star}, x \in M, w_{i}^{\prime} \in \boldsymbol{g}_{\mid x}^{\prime}$.

Proposition 4.2. (1) $H^{+\star}$ maps the invariant cross-sections into the invariant ones.

(2) $H^{+\star}$ restricted to the invariant cross-sections commutes with the differentials $\bar{\delta}^{\prime}$ and $\bar{\delta}$.

Proof. It is enough to prove the proposition in two cases of $H$ : of a strong homomorphism and of the canonical one. A very easy proof of (1) and (2) for the first case will be omitted. Consider now the canonical homomorphism $\mathrm{pr}_{2}$. Identify the vector bundles $f^{\star}(\boldsymbol{g} / \boldsymbol{h}) \cong f^{\star} \boldsymbol{g} / f^{\star} \boldsymbol{h}$. Then, of course, $H^{+\star} \Psi=f^{\star} \Psi$ and, by standard calculations, we get the following equality (cf. [K4, 2.3.2]):

$$
f^{\star}\left(\operatorname{ad}_{B, \boldsymbol{g}}\right)=\operatorname{ad}_{f^{\wedge} B, f^{\star} \boldsymbol{g}}
$$

which, together with $f^{\star}\left(\bigwedge^{k} T\right)=\bigwedge^{k}\left(f^{\star} T\right)$ for any representation $T$ (cf. [K4, 2.3.3]), yields

$$
f^{\star}\left(\operatorname{ad}_{B, \boldsymbol{g}}^{\wedge}\right)=f^{\star}\left(\bigwedge^{k}\left(\operatorname{ad}_{B, \boldsymbol{g}}\right)^{\natural}\right)=\bigwedge^{k}\left(\operatorname{ad}_{f^{\wedge} B, f^{\star} \boldsymbol{g}}\right)^{\natural}=\operatorname{ad}_{f^{\wedge} B, f^{\star} \boldsymbol{g}}^{\wedge}
$$

Proposition (2) needs now only standard calculations.

As a corollary we obtain that $H^{+\star}$ determines a homomorphism of algebras

$$
H^{+\#}: H(\boldsymbol{g}, B) \longrightarrow H\left(\boldsymbol{g}^{\prime}, B^{\prime}\right) .
$$

Proposition 4.3 (The functoriality of $\left.\Delta_{\#}\right)$. Let $\left(A^{\prime}, \lambda^{\prime}, B^{\prime}\right)$ and $(A, \lambda, B)$ be two $F S$ regular Lie algebroids over $\left(M^{\prime}, E^{\prime}\right)$ and $(M, E)$, respectively, and let

$$
H:\left(A^{\prime}, \lambda^{\prime}, B^{\prime}\right) \longrightarrow(A, \lambda, B)
$$

be a homomorphism between them over $f:\left(M^{\prime}, E^{\prime}\right) \rightarrow(M, E)$. Then the following diagram comutes: 


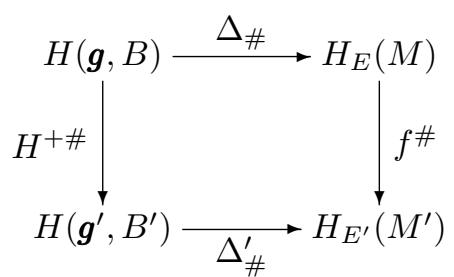

P r o o f. It is sufficient to show the commutativity of the diagram on the level of forms, that is, the equality: $\left(\gamma_{B^{\prime}}\right)_{\star}\left(f^{\star}\left(\Delta_{\star} \Psi\right)\right)=j^{\prime \star}\left(\omega^{\prime \wedge}\left(\bigwedge^{k} s^{\prime \star} \circ H^{+\star} \Psi\right)\right)$ for an invariant $\Psi$.

REMARK 2 . The problem of the equivalence of the characteristic homomorphisms $\Delta_{\#}$ and $\Delta_{\#}^{\prime}$ for $H$ being a homotopy equivalence is open. The problem reduces to the isomorphy of $H^{+\#}$. A definition of the homotopy between homomorphisms of FS-algebroids can be formulated in the spirit of the definition of the homotopy from [K7].

5. The homotopic independence of $\Delta_{\#}$ on a subalgebroid. Let $(A, \llbracket \cdot, \cdot \rrbracket, \gamma)$ be a given regular Lie algebroid with the Atiyah sequence $0 \rightarrow \boldsymbol{g} \hookrightarrow A \stackrel{\gamma}{\rightarrow} E \rightarrow 0$ and consider the Lie algebroid $\left(T \mathbb{R} \times A, \llbracket \cdot, \cdot \rrbracket^{\prime}\right.$, id $\left.\times \gamma\right)$, the product of the trivial Lie algebroid $T \mathbb{R}$ with $A$ (see $[\mathrm{H}-\mathrm{M}],[\mathrm{K} 7])$. Its Atiyah sequence is

$$
0 \longrightarrow 0 \times \boldsymbol{g} \longleftrightarrow T \mathbb{R} \times A \stackrel{\mathrm{id} \times \gamma}{\longrightarrow} T \mathbb{R} \times E \longrightarrow 0 .
$$

For the mapping $f_{t}: M \rightarrow \mathbb{R} \times M, \quad x \mapsto(t, x)$, take the pullback $f_{t}^{\wedge}(T \mathbb{R} \times A)$. Notice that $f_{t}^{\wedge}(T \mathbb{R} \times A)=\{(\gamma(w), 0, w) \in E \times(T \mathbb{R} \times A) ; w \in A\}$, and that the homomorphism $F_{t}: A \rightarrow T \mathbb{R} \times A, w \mapsto\left(\theta_{t}, w\right),\left(\theta_{t}\right.$ being the null tangent vector at $\left.t \in \mathbb{R}\right)$ of regular Lie algebroids (see the proof of Th. 4.3.1 in [K4]) is represented in the form of the canonical superposition

$$
F_{t}: A \stackrel{\bar{F}_{t}}{\longrightarrow} f_{t}^{\wedge}(T \mathbb{R} \times A) \stackrel{\mathrm{pr}_{2}}{\longrightarrow} T \mathbb{R} \times A
$$

(see $[\mathrm{K} 4, \mathrm{~s} .1 .1])$. It is not difficult to see that

$$
\bar{F}_{t}: A \longrightarrow f_{t}^{\wedge}(T \mathbb{R} \times A), w \longmapsto(\gamma(w), 0, w),
$$

is an isomorphism of regular Lie algebroids.

Definition 5.1. Two Lie subalgebroids $B_{0}, B_{1} \subset A$ (both over $(M, E)$ ) are said to be homotopic if there exists a Lie subalgebroid $B \subset T \mathbb{R} \times A$ over $(\mathbb{R} \times M, T \mathbb{R} \times E)$ such that the isomorphism $\bar{F}_{t}$ maps $B_{t}$ onto $f_{t}^{\wedge}(B)$ for $t=0,1$ (equivalently, if, for $v \in A$, we have: $\left.v \in B_{t} \Leftrightarrow\left(\theta_{t}, v\right) \in B\right)$. $B$ is called joining $B_{0}$ to $B_{1}$.

We compare the relation of homotopic subbundles of a principal bundle $P$ with the relation of homotopic subalgebroids of $A(P)$. Let $P=(P, \pi, M, G, \cdot)$ be a $G$-principal bundle over a manifold $M$. It determines a new $G$-principal bundle $\mathbb{R} \times P=(\mathbb{R} \times$ $P$, id $\left.\times \pi, \mathbb{R} \times M, G, \cdot^{\prime}\right)$ with the action $(t, z) \circ^{\prime} a=(t, z \cdot a)$. For an arbitrary $t \in \mathbb{R}$, the mapping $F_{t}: P \rightarrow f_{t}^{\star}(\mathbb{R} \times P), z \mapsto(\pi z,(t, z))$, is an isomorphism of $G$-principal bundles. Take a Lie subgroup $H \subset G$ (nonclosed and disconnected in general). Two $H$-reductions $P_{t} \subset P, t=0,1$, are said to be homotopic [K-T] if there exists an $H$-reduction $\bar{P} \subset \mathbb{R} \times P$ such that $F_{t}$ maps $P_{t}$ onto $f_{t}^{\star}(\bar{P})$ for $t=0,1 . \bar{P}$ is called joining $P_{0}$ to $P_{1}$. Notice that 
$\bar{P}$ is determined uniquely by the family of $H$-reductions $P_{t}=F_{t}^{-1}\left[f_{t}^{\star}(\bar{P})\right], t \in \mathbb{R}$ [which follows from the observation: $\left.z \in P_{t} \Leftrightarrow(t, z) \in \bar{P}\right]$.

If $H$ is closed and $P_{t}$ are defined by $C^{\infty}$ cross-sections $\sigma_{t}: M \rightarrow P / H$ for $t=0,1$, of the associated bundle $P / H \rightarrow M$, then, $P_{0}$ and $P_{1}$ are homotopic if and only if $\sigma_{0}$ and $\sigma_{1}$ are homotopic in the usual sense (via cross-sections, of course).

Proposition 5.2. If $P_{t} \stackrel{i_{t}}{\longrightarrow} P, t=0,1$, are homotopic $H$-reductions of $P$, then the Lie subalgebroids $B_{0}:=d\left(i_{0}\right)\left[A\left(P_{0}\right)\right]$ and $B_{1}:=d\left(i_{1}\right)\left[A\left(P_{1}\right)\right]$ of $A(P)$ are homotopic. The converse theorem is true provided that $P_{t}$ and $G$ are connected. More generally, if $A\left(P_{0}\right)$ and $A\left(P_{1}\right)$ are homotopic and $P_{0}$ and $P_{1}$ are connected [G can be disconnected], then there exists $a \in G$ such that $R_{a}\left[P_{0}\right]$ is homotopic to $P_{1}$.

Proof. Let $P_{0}, P_{1} \subset P$ be two $H$-reductions of $P$. Assume that they are homotopic, and that $\bar{P} \subset \mathbb{R} \times P$ is a joining $H$-reduction. Then $B:=\varphi[A(\bar{P})] \subset T \mathbb{R} \times A(P)$,

$$
\varphi: A(\mathbb{R} \times P)=T(\mathbb{R} \times P) / G \ni[(v, w)] \longmapsto(v,[w]) \in T \mathbb{R} \times T P / G=T \mathbb{R} \times A(P)
$$

being the canonical isomorphism, is a Lie algebroid joining $B_{0}$ to $B_{1}$. Indeed, one can easily see that $\bar{F}_{t}: A(P) \rightarrow f_{t}^{\wedge}(T \mathbb{R} \times A(P))$ equals the superposition

$$
A(P) \stackrel{d F_{t}}{\longrightarrow} A\left(f_{t}^{\star}(\mathbb{R} \times P)\right) \cong f_{t}^{\wedge}(A(\mathbb{R} \times P)) \cong f_{t}^{\wedge}(T \mathbb{R} \times A(P))
$$

and then maps $B_{t}$ onto $f_{t}^{\wedge}(B)$ for $t=0,1$.

Conversely, assume that the Lie subalgebroids $B_{0}$ and $B_{1}$ are homotopic, say, via a joining Lie subalgebroid $B$ of $T \mathbb{R} \times A(P)$. This means that $\bar{F}_{t}$ maps $B_{t}$ onto $f_{t}^{\wedge}(B)$ for $t=0,1$. Let $\bar{P} \subset \mathbb{R} \times P$ be the arbitrarily taken connected $H$-reduction corresponding to the Lie subalgebroid $\varphi^{-1}[B] \subset A(\mathbb{R} \times P)$, see Th. 1.1. Put $\tilde{P}_{t}:=F_{t}^{-1}\left[f_{t}^{\star}(\bar{P})\right], t \in \mathbb{R}$. By its construction, $\left\{\tilde{P}_{t}, t \in \mathbb{R}\right\}$ is a family of homotopic $H$-reductions. Of course, $P_{t}$ and $\tilde{P}_{t}$ are, for $t=0,1$, two $H$-reductions corresponding to the same Lie subalgebroid $B_{t}$. If $P_{t}$ is connected, then, since $P_{t}$ and $\tilde{P}_{t}$ are integral manifolds of the same $G$-right invariant distribution on $P$, we notice that $\tilde{P}_{t}=R_{g_{t}}\left[P_{t}\right]$ for a point $g_{t} \in G$. Therefore $R_{g_{0} g_{1}^{-1}}\left[P_{0}\right]$ is homotopic to $P_{1}$. If, additionally, $G$ is connected, $R_{g_{0} g_{1}^{-1}}\left[P_{0}\right]$ is homotopic to $P_{0}$. Thus $P_{0}$ and $P_{1}$ are homotopic then.

For further investigations, we fix

- a regular Lie algebroid $A=(A, \llbracket \cdot, \cdot \rrbracket, \gamma)$ over $(M, E)$,

- a flat connection $\lambda: E \rightarrow A$ in it,

- two Lie subalgebroids $B_{0}, B_{1} \subset A$, both over $(M, E)$, homotopic to each other via a joining Lie algebroid $B \subset T \mathbb{R} \times A$.

$\lambda$ determines a flat connection in $T \mathbb{R} \times A$ of the form id $\times \lambda: T \mathbb{R} \times E \rightarrow T \mathbb{R} \times A$. This implies that the triad

$$
(T \mathbb{R} \times A, \operatorname{id} \times \lambda, B)
$$

is an FS-regular Lie algebroid. Moreover,

$$
F_{t}:\left(A, \lambda, B_{t}\right) \longrightarrow(T \mathbb{R} \times A, \operatorname{id} \times \lambda, B)
$$

is a homomorphism of FS-regular Lie algebroids. 
Proposition 5.3. The characteristic homomorphisms $\Delta_{t \#}, t=0,1$, of FS-regular Lie algebroids $\left(A, \lambda, B_{t}\right)$ are related to each other by the commutativity of the following diagram:

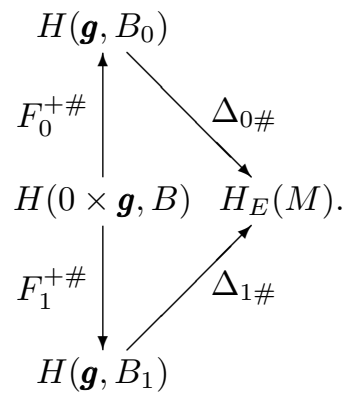

Proof. By the functoriality of the characteristic homomorphisms of FS-regular Lie algebroids, we get the commutative diagram

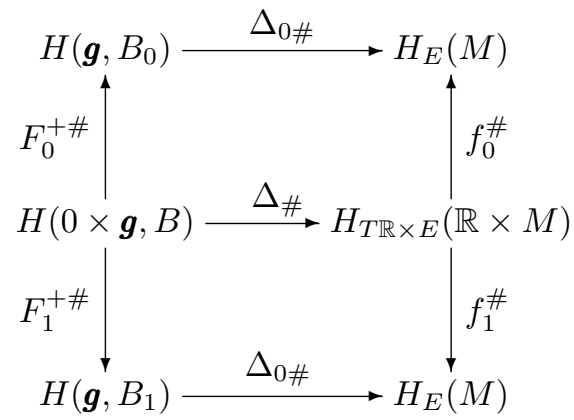

where $\Delta_{\#}$ is the characteristic homomorphism of (5.2). Since $f_{0}^{\#}=f_{1}^{\#}$ (see the proof of Th. 4.3.1 from [K4]) and $f_{0}^{\#}$ is an isomorphism (because $f_{0}$ and $f_{1}$ are homotopic in the category of foliated manifolds and each of them is a homotopy equivalence in this category) therefore $f_{1}^{\#}=\left(f_{0}^{\#}\right)^{-1}$, which implies our proposition.

Notice that if $F_{t}^{+\#}, t=0,1$, are isomorphisms, then $\Delta_{0 \#}$ and $\Delta_{1 \#}$ can be interpreted as equivalence homomorphisms in the sense of the following definition.

Definition 5.4. Let $B_{0}, B_{1} \subset A$ be two Lie subalgebroids of a flat regular Lie algebroid $A$ (all three over $(M, E)$ ). We say that the characteristic homomorphisms $\Delta_{t \#}$ : $H\left(\boldsymbol{g}, B_{t}\right) \rightarrow H_{E}(M), t=0,1$, corresponding to $B_{0}$ and $B_{1}$, respectively, are equivalent if there exists an isomorphism of algebras $\alpha: H\left(\boldsymbol{g}, B_{0}\right) \stackrel{\cong}{\longrightarrow} H\left(\boldsymbol{g}, B_{1}\right)$ such that $\Delta_{0 \#}=$ $\Delta_{1 \#} \circ \alpha$.

THEOREM 5.5. If $B_{0}$ and $B_{1}$ are homotopic, then $\Delta_{0 \#}$ and $\Delta_{1 \#}$ are equivalent.

Proof. Recall that $F_{t}=\mathrm{pr}_{2} \circ \bar{F}_{t}$, see (5.1). $\bar{F}_{t}$ is an isomorphism of FS-regular Lie algebroids, therefore

$$
\bar{F}_{t}^{+\#}: H\left(f_{t}^{\star}(0 \times \boldsymbol{g}), f_{t}^{\wedge} B\right) \stackrel{\cong}{\longrightarrow} H\left(\boldsymbol{g}, B_{t}\right)
$$

is an isomorphism of algebras. It remains to consider the homomorphism 


$$
\operatorname{pr}_{2}^{+\#}: H(0 \times \boldsymbol{g}, B) \rightarrow H\left(f_{t}^{\star}(0 \times \boldsymbol{g}), f_{t}^{\wedge} B\right) .
$$

Identifying (via the canonical isomorphism) the vector bundles

$$
f_{t}^{\star}(0 \times \boldsymbol{g}) / f_{t}^{\star} \boldsymbol{h} \cong f_{t}^{\star}(0 \times \boldsymbol{g} / \boldsymbol{h}),
$$

we get (cf. the proof of Prop.5.2.1 [K4])

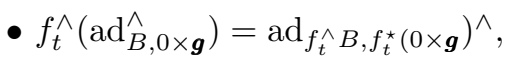

- $\operatorname{pr}_{2}^{+\#}: \bigoplus_{k>0}\left(\operatorname{Sec} \bigwedge^{k}(0 \times \boldsymbol{g} / \boldsymbol{h})^{\star}\right)_{I^{0}} \rightarrow \bigoplus_{k>0}\left(\operatorname{Sec} \bigwedge^{k} f_{t}^{\star}(0 \times \boldsymbol{g} / \boldsymbol{h})^{\star}\right)_{I^{0}}$ is the usual pullback $\Psi \mapsto f_{t}^{\star} \bar{\Psi}$.

Theorem 5.5 follows now from Theorem 1.3.

6. Comparison with the characteristic classes of a flat principal fibre bundle. Given:

(a) a $G$-principal fibre bundle $P=(P, \pi, M, G, \cdot)$,

(b) a flat connection in $P$ with connection form $\omega$,

(c) a closed Lie subgroup $H \subset G$ and an $H$-reduction $P^{\prime} \subset P$,

let $\mathfrak{g}$ and $\mathfrak{h}$ denote the Lie algebras of $G$ and $H$, respectively. Of course, $i: P^{\prime} \hookrightarrow P$ is an $(H \hookrightarrow G)$-homomorphism of principal bundles and its differential $d i: A\left(P^{\prime}\right) \rightarrow A(P)$, see [K3], [M1], is a monomorphism of the corresponding transitive Lie algebroids

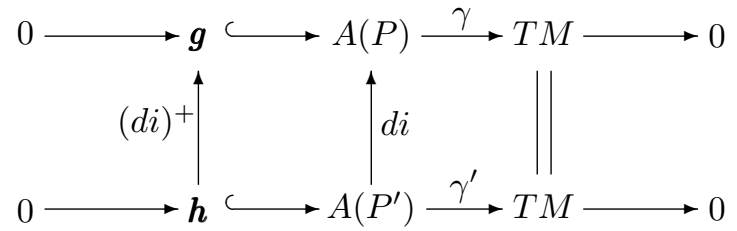

Identify $A\left(P^{\prime}\right)$ with $\operatorname{Im}(d i)$ and $\boldsymbol{h}$ with $\operatorname{Im}(d i)^{+}$. Then, for each $z \in P_{\mid x}^{\prime}$, the isomorphism $\hat{z}: \mathfrak{g} \rightarrow \boldsymbol{g}_{\mid x}, v \mapsto\left[A_{z \star v}\right]\left(A_{z}: G \rightarrow P, a \mapsto z a\right)$, see [K4, s.5.1], maps $\mathfrak{h}$ onto $\boldsymbol{h}_{\mid x}$ and determines an isomorphism $[\hat{z}]: \mathfrak{g} / \mathfrak{h} \cong(\boldsymbol{g} / \boldsymbol{h})_{\mid x}$. It is worth recalling that $\hat{z}$ is an isomorphism of Lie algebras provided that $\mathfrak{g}$ is the right Lie algebra of $G$, see [K3], [K4].

According to section 3, we have a representation $\operatorname{ad}_{A\left(P^{\prime}\right), \boldsymbol{g}}: A\left(P^{\prime}\right) \rightarrow A(\boldsymbol{g} / \boldsymbol{h})$ such that $\mathfrak{L}_{\mathrm{ad}_{A\left(P^{\prime}\right), \boldsymbol{g}} \circ \xi}([\nu])=[\llbracket \xi, \nu \rrbracket], \xi \in \operatorname{Sec} A\left(P^{\prime}\right), \nu \in \operatorname{Sec} \boldsymbol{g}$, and the representation induced by it $\operatorname{ad}_{A\left(P^{\prime}\right), \boldsymbol{g}}^{\wedge}: A\left(P^{\prime}\right) \rightarrow A\left(\bigwedge^{k}(\boldsymbol{g} / \boldsymbol{h})^{\star}\right)$. Consider also the induced representation $\operatorname{Ad}_{P^{\prime}, \boldsymbol{g}}$ of the principal bundle $P^{\prime}$ on the $\mathfrak{g} / \mathfrak{h}$-vector bundle $\boldsymbol{g} / \boldsymbol{h}$, defined by $\operatorname{Ad}_{P^{\prime}, \boldsymbol{g}}: P^{\prime} \rightarrow$ $L(\boldsymbol{g} / \boldsymbol{h}), z \mapsto[\hat{z}]$, and the representation $\operatorname{Ad}_{P^{\prime}, \boldsymbol{g}}^{\wedge}: P^{\prime} \rightarrow L\left(\bigwedge^{k}(\boldsymbol{g} / \boldsymbol{h})^{\star}\right)$ induced by it (cf. $[\mathrm{K} 4,5.3 .2])$. By the same argument as in the proof of Th. 5.4.3 in [K4], to see that $\operatorname{ad}_{A\left(P^{\prime}\right), \boldsymbol{g}}$ is the differential of $\operatorname{Ad}_{P^{\prime}, \boldsymbol{g}}$, we must only notice an analogous fact concerning the representations of Lie algebras and of Lie groups: $\mathfrak{h} \rightarrow \operatorname{End}(g / h), v \mapsto\left[\operatorname{ad}_{\mathfrak{g}}(v)\right]$, and $H \rightarrow G L(\mathfrak{g} / \mathfrak{h}), h \mapsto\left[\operatorname{Ad}_{G}(h)\right]$. Hence, $\operatorname{ad}_{A\left(P^{\prime}\right), \boldsymbol{g}}^{\wedge}$ is the differential of $\operatorname{Ad}_{P^{\prime}, \boldsymbol{g}}^{\wedge}$. Therefore, according to [K4, Props 5.5.2-3], we have a monomorphism

$$
\kappa:\left(\bigwedge(\mathfrak{g} / \mathfrak{h})^{\star}\right)_{I} \stackrel{\cong}{\longrightarrow} \bigoplus_{k \geq 0}\left(\operatorname{Sec} \bigwedge^{k}(\boldsymbol{g} / \boldsymbol{h})^{\star}\right)_{I} \subset \bigoplus_{k \geq 0}\left(\operatorname{Sec} \bigwedge^{k}(\boldsymbol{g} / \boldsymbol{h})^{\star}\right)_{I^{0}}
$$


defined by the formula $\kappa(\psi)(x)=\operatorname{Ad}_{P^{\prime}, \boldsymbol{g}}^{\wedge}(z)(\psi), z \in P_{\mid x}^{\prime}$, and which is an isomorphism when $P^{\prime}$ is connected. It is necessary to verify that $\kappa$ commutes with the differentials $d^{H}$ and $\bar{\delta}$ acting on the spaces $\left(\bigwedge(\mathfrak{g} / \mathfrak{h})^{\star}\right)_{I}$ and $\bigoplus_{k \geq 0}\left(\operatorname{Sec} \bigwedge^{k}(\boldsymbol{g} / \boldsymbol{h})^{\star}\right)_{I^{0}}$, respectively (notice that the spaces of cohomology of these are domains of the characteristic homomorphisms). The differential $\bar{\delta}$ in $\bigoplus_{k>0}\left(\operatorname{Sec} \bigwedge^{k}(\boldsymbol{g} / \boldsymbol{h})^{\star}\right)_{I^{0}}$ is defined in section 3 above, whereas, the differential $d^{H}$ in $\left(\bigwedge(\mathfrak{g} / \mathfrak{h})^{\star}\right)_{I}$ must be defined by the formula

$$
\left\langle d^{H}(\psi),\left[w_{1}\right] \wedge \ldots \wedge\left[w_{k}\right]\right\rangle=\sum_{i<j}(-1)^{i+j}\left\langle\psi,\left[\left[w_{i}, w_{j}\right]\right] \wedge\left[w_{1}\right] \wedge \ldots \hat{i} \ldots \hat{j} \ldots\right\rangle,
$$

$w_{1}, \ldots, w_{k} \in \mathfrak{g}$; here $\left[w_{i}, w_{j}\right]$ is the bracket in the left Lie algebra of $G$ [we get it following the fact that this differential must be the one for which the canonical isomorphism $G_{\Omega^{\star}(G / H)} \cong\left(\bigwedge(\mathfrak{g} / \mathfrak{h})^{\star}\right)_{I}$ (also $\left.\left(\bigwedge \mathfrak{g}^{\star}\right)^{H} \cong\left(\bigwedge(\mathfrak{g} / \mathfrak{h})^{\star}\right)_{I}\right)$ is an isomorphism of DG-algebras, see [K-T]]. The equality $\bar{\delta} \circ \kappa^{k}=\kappa^{k+1} \circ d^{H}$ may now be obtained immediately.

Theorem 6.1. The characteristic homomorphisms $\Delta_{\#}: H(\mathfrak{g}, H) \rightarrow H_{d R}(M)$ of the triad $\left(P, P^{\prime}, \omega\right)($ see $[\mathrm{K}-\mathrm{T}])$ and $\Delta_{\#}: H\left(\boldsymbol{g}, A\left(P^{\prime}\right)\right) \rightarrow H_{d R}(M)$ of the FS-transitive Lie algebroid $\left(A(P), \lambda, A\left(P^{\prime}\right)\right)$ ( $\lambda$ corresponds to $\omega$ ) are related by the following commutative diagram:

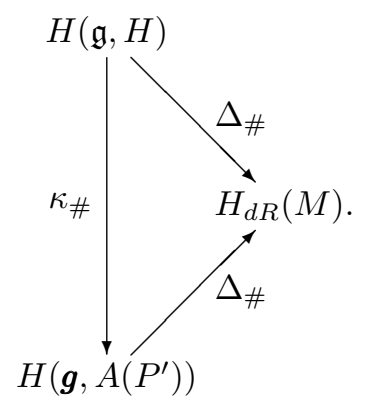

Proof. We prove the commutativity on the level of forms. Consider the diagram

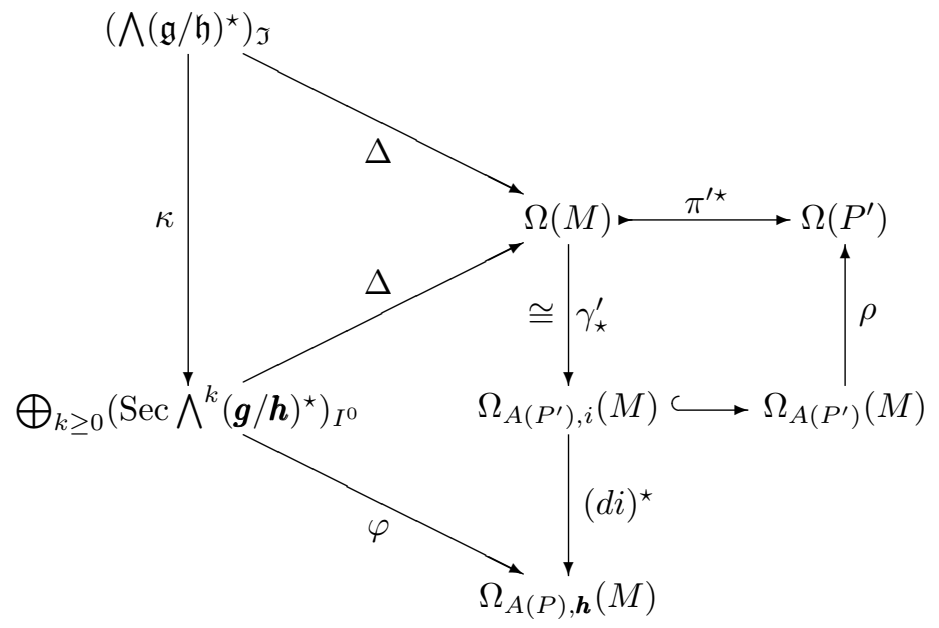

in which 
- $\varphi(\Psi)=\left(\omega^{A}\right)^{\wedge}\left(\bigwedge^{k} \boldsymbol{s}^{\star} \circ \Psi\right)=\frac{1}{k !} \cdot\left\langle\bigwedge^{k} \boldsymbol{s}^{\star} \circ \Psi, \omega^{A} \wedge \ldots \wedge \omega^{A}\right\rangle, \omega^{A}: A(P) \rightarrow \boldsymbol{g}$ being the connection form corresponding to $\lambda$,

- $\Omega_{A(P), \boldsymbol{h}}(M)$ denotes the space of $\boldsymbol{h}$-horizontal forms on $A(P)$,

- $\rho$ maps real forms on $A\left(P^{\prime}\right)$ into right-invariant forms on $P^{\prime}, \Theta \mapsto \tilde{\Theta}$ for $\tilde{\Theta}$ (see $[\mathrm{K} 3])$.

We recall that, for $\psi \in\left(\bigwedge^{k}(\mathfrak{g} / \mathfrak{h})^{\star}\right)_{I}$, the form $\Delta(\psi) \in \Omega^{k}(M)$ is defined uniquely in such a way that $\pi^{\prime \star}(\Delta(\psi))=\frac{1}{k !} \cdot i^{\star}\left\langle\bigwedge^{k} s^{\star}(\psi), \omega \wedge \ldots \wedge \omega\right\rangle$ where $i: P^{\prime} \hookrightarrow P$, whereas $s: \mathfrak{g} \rightarrow \mathfrak{g} / \mathfrak{h}$ and $\pi^{\prime}: P^{\prime} \rightarrow M$ are the canonical projections. On the other hand, $\Delta(\Psi)$ for $\Psi \in\left(\operatorname{Sec} \bigwedge^{k}(\boldsymbol{g} / \boldsymbol{h})^{\star}\right)_{I^{0}}$ is given as a form for which $\gamma_{\star}^{\prime}(\Delta(\Psi))=(d i)^{\star}(\varphi(\Psi))$. Therefore, to end the proof, we need to prove the equality $i^{\star}\left\langle\bigwedge^{k} s^{\star}(\psi), \omega \wedge \ldots \wedge \omega\right\rangle=$ $\rho \circ(d i)^{\star}\left(\left\langle\bigwedge^{k} \boldsymbol{s}^{\star}(\kappa \psi), \omega^{A} \wedge \ldots \wedge \omega^{A}\right\rangle\right)$ only (very easy calculations are omitted).

7. The tangential characteristic classes of a partially flat principal bundle. Consider now Ex. 3.1(2), i.e. a triple $(A, B, \lambda)$ consisting of a transitive Lie algebroid $A$ on $M$, a transitive Lie subalgebroid $B$ of $A$ and a partially flat connection $\lambda$ in $A$, namely, flat over a given involutive distribution $F \subset T M$. The characteristic homomorphism $\Delta_{\#}^{F}: H\left(\boldsymbol{g}, B^{F}\right) \rightarrow H_{F}(M)$ of the FS-regular Lie algebroid $\left(A^{F}, B^{F}, \lambda \mid F\right)$ will be called the tangential characteristic homomorphism of the system $(A, B, \lambda)$, and the cohomology classes from its image the tangential characteristic classes of the system $(A, B, \lambda)$.

Let now the system $\left(P, P^{\prime}, \omega\right)$ be given as in Ex. 3.1(3). It determines the FS-regular Lie algebroid $\left(A(P)^{F}, A\left(P^{\prime}\right)^{F}, \lambda \mid F\right)$, and via this a characteristic homomorphism

$$
\Delta_{\#}^{F}: H\left(\boldsymbol{g}, A\left(P^{\prime}\right)^{F}\right) \longrightarrow H_{F}(M),
$$

called the characteristic homomorphism of the system $\left(P, P^{\prime}, \omega\right)$. The cohomology classes from the image of $\Delta_{\#}^{F}$ should be called the tangential characteristic classes of the system $\left(P, P^{\prime}, \omega\right)$. By construction, they measure the independence of $\omega$ and $P^{\prime}$ - exactly the same as the exotic characteristic classes of a partially flat principal bundle [K-T].

REMARK 3. The problem of comparing these two systems of characteristic classes is open.

8. The case of a TC-foliation. This section is a continuation of Example 3.1(4). One can prove the following two propositions.

Proposition 8.1. The Lie algebroid $T \mathbb{R} \times A(M, F)$ is canonically isomorphic to the Lie algebroid $A(\mathbb{R} \times M, \tilde{F})$ of the $T C$-foliation $(\mathbb{R} \times M, \tilde{F}):=\left(\mathbb{R} \times E_{d}\right) \times(M, F)$ which is the product of the discrete foliation $E_{d}$ of $\mathbb{R}$ and the given foliation $(M, F)$.

Let $B_{0}, B_{1} \subset A(M, F)$ be two Lie subalgebroids of $A(M, F)$, and $\tilde{B}_{0}, \tilde{B}_{1} \subset T M$ the corresponding involutive subbundles (see Ex. 3.1(4)). A simple consequence of the definitions and Proposition 8.1 is the following

Proposition 8.2. $B_{0}$ and $B_{1}$ are homotopic if and only if there exists an involutive subbundle $\bar{B} \subset T(\mathbb{R} \times M)=T \mathbb{R} \times T M$ such that

(1) $0 \times F \subset \bar{B}$,

(2) $\bar{B}+\left(0 \times F_{b}\right)=T \mathbb{R} \times T M$, 
(3) $v \in \bar{B}_{t} \Leftrightarrow\left(\theta_{t}, v\right) \in \bar{B} \quad$ for $t=0,1$,

(4) the Lie algebra $\operatorname{Sec} \bar{B} \cap L(\mathbb{R} \times M, \tilde{F})$ generates at each point $(t, x) \in \mathbb{R} \times M$ the entire space $\bar{B}_{\mid(t, x)}$.

Here we pass to the Lie algebroid $A(G ; H)$ of the TC-foliation $\mathcal{H}$ of a connected Lie group $G$ by left cosets of a connected nonclosed Lie subgroup $H \subset G$. Let $\mathfrak{h}, \overline{\mathfrak{h}}, \mathfrak{g}$ denote, as usual, the Lie algebras of $H, \bar{H}$ and $G$, respectively. By the same argument as in Example 7.4.7 from [K4] we prove

Lemma 8.3. Let $\mathfrak{b} \subset \mathfrak{g}$ be a Lie subalgebra such that

$$
\mathfrak{h} \subset \mathfrak{b}, \quad \overline{\mathfrak{h}}+\mathfrak{b}=\mathfrak{g} ;
$$

then the $G$-left-invariant distribution $\bar{B}_{\mathfrak{b}} \subset T G$ determined by $\mathfrak{b}$ [i.e. the one tangent to the foliation $\{g \cdot B ; g \in G\}, B$ being the connected Lie subgroup with the Lie algebra $\mathfrak{b}$ ] fulfils conditions (1), (2), (3') from Example 3.1(4), giving at the same time a transitive Lie subalgebroid of $A(G ; H)$.

It seems to be interesting that $\mathfrak{b}$ can be interpreted as a "connection", but in another Lie algebroid. Namely, let $H_{1}$ be the connected Lie subgroup of $G$ whose Lie algebra equals $\mathfrak{b} \cap \overline{\mathfrak{h}}$. Of course, $\mathfrak{h} \subset \mathfrak{b} \cap \overline{\mathfrak{h}} \subset \overline{\mathfrak{h}}$, therefore $H \subset H_{1} \subset \bar{H}$, hence $\bar{H}_{1}=\bar{H}$. Then, it is clear (see [K4, Ex.8.4.7]) that $\bar{B}_{\mathfrak{b}}$ is an $\mathcal{F}$-connection where $\mathcal{F}$ is the foliation of left cosets of $H_{1}$ in $G$.

Lemma 8.4. Let $\mathfrak{c} \subset \mathfrak{g}$ be a Lie subalgebra such that

$$
\mathfrak{c} \cap \overline{\mathfrak{h}}=\mathfrak{h}, \quad \mathfrak{c}+\overline{\mathfrak{h}}=\mathfrak{g} ;
$$

then the $G$-left distribution $C_{\mathfrak{c}}$ determined by $\mathfrak{c}$ is $C^{\infty}$ completely integrable and fulfils (a), (b), (c') from Example 3.1(4), giving at the same time a flat connection in $A(G ; H)$.

Seeing that the foliation $\{\mathbb{R} \times G, \overline{\mathcal{H}}\}=\left\{\mathbb{R}, E_{d}\right\} \times\{G, \mathcal{H}\}$ is equal to the foliation of the Lie group $\mathbb{R} \times G$ by left cosets of a Lie subgroup $\Theta \times H, \Theta$ being the null Lie subgroup of $\mathbb{R}$, we get

LEMma 8.5. The Lie algebroid $T \mathbb{R} \times A(G ; H)$ is isomorphic to the Lie algebroid $A(\mathbb{R} \times$ $G ; \Theta \times H)$. Condition (4) from Theorem 8.2 above is equivalent to

(4') $\bar{B}$ is $\Theta \times \bar{H}$-right-invariant.

Now, we calculate the characteristic homomorphism of the FS-transitive Lie algebroid $(A(G ; H), B, \lambda)$ in which

- $B=B_{\mathfrak{b}}$ for a Lie subalgebra $\mathfrak{b}$ fulfilling (8.1),

- $\lambda$ is the flat connection determined by a Lie subalgebra $\mathfrak{c}$ fulfilling (8.2). (According to $[\mathrm{K} 5]$ for such a Lie subalgebra $\mathfrak{c}$ to exist, $\pi_{1}(G)$ must be infinite).

Recall that $[\mathrm{K} 4,8.2 .4] \varphi: G / \bar{H} \times \overline{\mathfrak{h}} / \mathfrak{h} \rightarrow \boldsymbol{g},(\bar{g},[w]) \mapsto\left[\bar{X}_{w}(g)\right], g \in \pi_{b}^{-1}(\bar{g})$, is a global trivialization of the Lie algebra bundle $\boldsymbol{g}$ of $A(G ; H)$, and that the typical fibre $\overline{\mathfrak{h}} / \mathfrak{h}$ of this bundle is an abelian Lie algebra $\left[\bar{X}_{w}\right.$ stands for the left-invariant vector field on $G$ generated by a vector $w][K 4,8.1 .3]$. The equalities $\boldsymbol{h}=\boldsymbol{g} \cap B$ and $\operatorname{dim}(\overline{\mathfrak{h}} \cap \mathfrak{b} / \mathfrak{h})=\operatorname{rank} \boldsymbol{h}$ yield that $\varphi$ induces a global trivialization $\varphi^{1}: G / \bar{H} \times(\overline{\mathfrak{h}} \cap \mathfrak{b}) / \mathfrak{h} \rightarrow \boldsymbol{h}$. Next, $\varphi$ and $\varphi^{1}$ give a global trivialization $\varphi^{2}: G / \bar{H} \times \overline{\mathfrak{h}} /(\overline{\mathfrak{h}} \cap \mathfrak{b}) \rightarrow \boldsymbol{g} / \boldsymbol{h}$. Using $\varphi^{2}$, we can modify any 
cross-section $\nu \in \operatorname{Sec} \boldsymbol{g} / \boldsymbol{h}$ to the $\overline{\mathfrak{h}} /(\overline{\mathfrak{h}} \cap \mathfrak{b})$-valued function $\bar{\nu}: G / \bar{H} \rightarrow \overline{\mathfrak{h}} /(\overline{\mathfrak{h}} \cap \mathfrak{b})$, and, analogously, any cross-section $\Psi \in \operatorname{Sec} \bigwedge^{k}(\boldsymbol{g} / \boldsymbol{h})^{\star}$ to the function $\bar{\Psi}: G / \bar{H} \rightarrow \bigwedge^{k}(\overline{\mathfrak{h}} /(\overline{\mathfrak{h}} \cap$ $\mathfrak{b}))^{\star}$. Analogously to the proof of Prop. 7.4.1 from $[K 4]$ we obtain the following

Proposition 8.6. $\Psi$ is invariant if and only if $\bar{\Psi}$ is constant.

In the space $\bigoplus_{k \geq 0}\left(\operatorname{Sec} \bigwedge^{k}(\boldsymbol{g} / \boldsymbol{h})^{\star}\right)_{I^{0}}$ the differential $\bar{\delta}$ defined by (3.1) works. We can carry $\bar{\delta}$ over to the space $\bigwedge(\overline{\mathfrak{h}} /(\overline{\mathfrak{h}} \cap \mathfrak{b}))^{\star}$ and obtain a differential $\overline{\bar{\delta}}$.

Proposition 8.7. $\overline{\bar{\delta}}=0[$ hence $\bar{\delta}=0]$.

Proof. Take $\hat{\Psi} \in \bigwedge^{k}(\overline{\mathfrak{h}} /(\overline{\mathfrak{h}} \cap \mathfrak{b}))^{\star}$ and let $\Psi \in\left(\operatorname{Sec} \bigwedge^{k}(\boldsymbol{g} / \boldsymbol{h})^{\star}\right)_{I^{\circ}}$ correspond to $\hat{\Psi}$ (i.e. $\hat{\Psi}=\bar{\Psi}(\bar{e})=\bar{\Psi}(\bar{g}))$. For $w_{1}, \ldots, w_{k} \in \overline{\mathfrak{h}}$, we have

$$
\left\langle\hat{\Psi},\left[w_{1}\right] \wedge \ldots \wedge\left[w_{k}\right]\right\rangle=\left\langle\Psi(\bar{g}),\left[\varphi_{\bar{g}}\left(\left[w_{1}\right]\right)\right] \wedge \ldots \wedge\left[\varphi_{\bar{g}}\left(\left[w_{k}\right]\right)\right]\right\rangle
$$

and then (see $[\mathrm{K} 4],[\mathrm{MO} 2])$ for $w_{0}, \ldots, w_{k} \in \overline{\mathfrak{h}}$,

$$
\begin{aligned}
& \left\langle\overline{\bar{\delta}} \hat{\Psi},\left[w_{0}\right] \wedge \ldots \wedge\left[w_{k}\right]\right\rangle \\
= & \left\langle(\bar{\delta} \Psi)^{\wedge},\left[w_{0}\right] \wedge \ldots \wedge\left[w_{k}\right]\right\rangle \\
= & \left\langle(\bar{\delta} \Psi),\left[c\left(\bar{X}_{w_{0}}\right)\right] \wedge \ldots \wedge\left[c\left(\bar{X}_{w_{k}}\right)\right]\right\rangle(\bar{g}) \\
= & \left.-\sum_{i<j}(-1)^{i+j}\left\langle\Psi,\left[\llbracket c\left(\bar{X}_{w_{i}}\right), c\left(\bar{X}_{w_{j}}\right)\right]\right] \wedge\left[c\left(\bar{X}_{w_{0}}\right)\right] \wedge \ldots \hat{i} \ldots \hat{j} \ldots\right\rangle(\bar{g}) \\
= & -\sum_{i<j}(-1)^{i+j}\left\langle\Psi,\left[c\left(\bar{X}_{\left[w_{i}, w_{j}\right]}\right)\right] \wedge \ldots \hat{i} \ldots \hat{j} \ldots\right\rangle(\bar{g}) \\
= & -\sum_{i<j}(-1)^{i+j}\left\langle\hat{\Psi},\left[\left[w_{i}, w_{j}\right]\right] \wedge \ldots \hat{i} \ldots \hat{j} \ldots\right\rangle=0
\end{aligned}
$$

because $\left[w_{i}, w_{j}\right] \in \mathfrak{h} \subset \mathfrak{b} \cap \overline{\mathfrak{h}}[\overline{\mathfrak{h}} / \mathfrak{h}$ is abelian!].

As a corollary we obtain an isomorphism of algebras

$$
H(\boldsymbol{g}, B) \cong H\left(\bigwedge(\overline{\mathfrak{h}} /(\overline{\mathfrak{h}} \cap \mathfrak{b}))^{\star}, 0\right)=\bigwedge(\overline{\mathfrak{h}} /(\overline{\mathfrak{h}} \cap \mathfrak{b}))^{\star}
$$

and the fact that the forms from the image of the characteristic homomorphism, $\operatorname{Im} \Delta_{\star}$, are closed.

Take into account the connection $\lambda$ determined by $\mathfrak{c}$. Let $\omega$ be its connection form. Conditions (8.2) determine a decomposition $\mathfrak{g} / \mathfrak{h}=\overline{\mathfrak{h}} / \mathfrak{h} \oplus \mathfrak{c} / \mathfrak{h}$. Define $\omega_{0}: \mathfrak{g} \rightarrow \overline{\mathfrak{h}} / \mathfrak{h}$ as the linear mapping which is the superposition $\omega_{0}: \mathfrak{g} \rightarrow \mathfrak{g} / \mathfrak{h}=\overline{\mathfrak{h}} / \mathfrak{h} \oplus \mathfrak{c} / \mathfrak{h} \stackrel{\mathrm{pr}_{1}}{\longrightarrow} \overline{\mathfrak{h}} / \mathfrak{h}$. Take also the canonical linear homomorphism $\rho: \overline{\mathfrak{h}} / \mathfrak{h} \rightarrow \overline{\mathfrak{h}} /(\overline{\mathfrak{h}} \cap \mathfrak{b})$ and put $\omega_{1}=\rho \circ \omega_{o}: \mathfrak{g} \rightarrow \overline{\mathfrak{h}} /(\overline{\mathfrak{h}} \cap \mathfrak{b})$.

Lemma 8.8. The form $\Delta_{\star}(\Psi)$ for $\Psi \in\left(\operatorname{Sec} \bigwedge^{k}(\boldsymbol{g} / \boldsymbol{h})^{\star}\right)_{I^{0}}$ is a $G$-left-invariant form on $G / \bar{H}$ such that its value $\Delta_{\star}(\Psi)_{\bar{e}} \in \bigwedge^{k}(\mathfrak{g} / \overline{\mathfrak{h}})^{\star}$ at $\bar{e}$ is equal to

$$
\left\langle\Delta_{\star}(\Psi)_{\bar{e}},\left[w_{1}\right] \wedge \ldots \wedge\left[w_{k}\right]\right\rangle=\left\langle\hat{\Psi}, \omega_{1}\left(\tilde{w}_{1}\right) \wedge \ldots \wedge \omega_{1}\left(\tilde{w}_{k}\right)\right\rangle
$$

where $\tilde{w}_{i} \in \mathfrak{b}$ are vectors such that $\left[\tilde{w}_{i}\right]=\left[w_{i}\right] \in \mathfrak{g} / \overline{\mathfrak{h}}$ (notice that $\mathfrak{b} \hookrightarrow \mathfrak{g} \rightarrow \mathfrak{g} / \overline{\mathfrak{h}}$ is an epimorphism). 
Lemma 8.9. Consider the $D G$-algebra $\left(\bigwedge(\mathfrak{g} / \overline{\mathfrak{h}})^{\star}\right)_{I}$ of vectors invariant with respect to the adjoint representation $\mathrm{Ad}^{\perp}: \bar{H} \rightarrow G L\left(\bigwedge(\mathfrak{g} / \overline{\mathfrak{h}})^{\star}\right)$, see $[G-H-V$, Vol. II, Prop. XI]. The forms from $\operatorname{Im} \hat{\Delta}_{\star}$ are closed, where

$$
\hat{\Delta}_{\star}: \bigwedge(\overline{\mathfrak{h}} /(\overline{\mathfrak{h}} \cap \mathfrak{b}))^{\star} \longrightarrow\left(\bigwedge(\mathfrak{g} / \overline{\mathfrak{h}})^{\star}\right)_{I}
$$

is defined by the equality ( $\tilde{w}_{i}$ as above)

$$
\left\langle\hat{\Delta}_{\star}(\bar{\Psi}),\left[w_{1}\right] \wedge \ldots \wedge\left[w_{k}\right]\right\rangle=\left\langle\bar{\Psi}, \omega_{1}\left(\tilde{w}_{1}\right) \wedge \ldots \wedge \omega_{1}\left(\tilde{w}_{k}\right)\right\rangle .
$$

From the above we obtain the fundamental commutative diagram

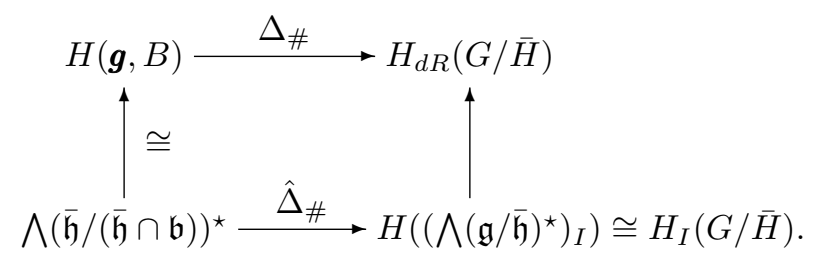

If $G$ is compact, then the right arrow is an isomorphism.

THEOREM 8.10. $\hat{\Delta}_{\#}$ is trivial if and only if $\mathfrak{c} \subset \mathfrak{b}$.

Pro of. Let $\mathfrak{c} \subset \mathfrak{b}$. The epimorphy of $\mathfrak{b} \hookrightarrow \mathfrak{g} \rightarrow \mathfrak{g} / \overline{\mathfrak{h}}$ implies that it is sufficient to show the equality $\omega_{1}(w)=0$ for $w \in \mathfrak{b}$. For this purpose, take an arbitrary point $w \in \mathfrak{b}$ and write $w=w_{1}+w_{2}$ for $w_{1} \in \overline{\mathfrak{h}}$ and $w_{2} \in \mathfrak{c}$. Then $w_{1}=w-w_{2} \in \mathfrak{b}$, so $\omega_{1}(w)=\rho\left(w_{1}\right)=0$.

Assume now $\mathfrak{c} \subset \mathfrak{b}$. Take $w \in \mathfrak{c} \backslash \mathfrak{b}$ and let $\tilde{w} \in \mathfrak{b}$ be a vector such that $[w]=[\tilde{w}]$ in $\mathfrak{g} / \overline{\mathfrak{h}}$. Of course, $\tilde{w}-w \in \overline{\mathfrak{h}} \backslash(\overline{\mathfrak{h}} \cap \mathfrak{b})$ and $\tilde{w}=(\tilde{w}-w)+w \in \overline{\mathfrak{h}}+\mathfrak{c}$. Take a covector $\bar{\Psi} \in(\overline{\mathfrak{h}} /(\overline{\mathfrak{h}} \cap \mathfrak{b}))^{\star}$ such that $\hat{\Psi}(\tilde{w}-w) \neq 0$. Then

$$
\hat{\Delta}_{\star}(\hat{\Psi})([w])=\left\langle\hat{\Psi}, \omega_{1}(\tilde{w})\right\rangle=\langle\hat{\Psi},[\tilde{w}-w]\rangle \neq 0 .
$$

Since $Z\left((\mathfrak{g} / \overline{\mathfrak{h}})^{\star}\right)_{I} \rightarrow H^{1}\left(\left(\bigwedge(\mathfrak{g} / \overline{\mathfrak{h}})^{\star}\right)_{I}\right)$ is a monomorphism, we obtain $\hat{\Delta}_{\#}(\hat{\Psi}) \neq 0$.

Then, for compact $G$, each case $\mathfrak{c} \not \mathfrak{b}$ is the source of the nontrivial characteristic homomorphism of a FS-transitive Lie algebroid on the ground of TC-foliations of left cosets.

\section{References}

[A-M] R. Almeida and P. Molino, Suites d'Atiyah et feuilletages transversalement complets, C. R. Acad. Sci. Paris Sér. I Math. 300 (1985).

[B] B. Balcerzak, Lie algebroid of a vector bundle, in preparation.

[C-D-W] A. Coste, P. Dazord and A. Weinstein, Groupoïdes symplectiques, Publ. Dep. Math. Université de Lyon 1, 2/A (1987).

[G] W. Greub, Multilinear algebra, Springer-Verlag, 1967.

[G-H-V] W. Greub, S. Halperin and R. Vanstone, Connections, Curvature, and Cohomology, Vol. II, 1973, Vol. III, 1976.

[H-M] Ph. Higgins and K. Mackenzie, Algebraic construction in the category of Lie algebroids, Journal of Algebra 129 (1990), 194-230. 
[K-T] F. Kamber and Ph. Tondeur, Foliated Bundles and Characteristic Classes, Lectures Notes in Mathematics 493, Springer-Verlag 1975.

[K1] J. Kubarski, Pradines-type groupoids over foliations; cohomology, connections and the Chern-Weil homomorphism, Technical University, Institute of Mathematics, Preprint 2, August 1986.

[K2] -, Exponential mapping for Lie groupoids. Applications, Colloquium Mathematicum, 54 (1987), 39-48.

[K3] _, Lie algebroid of a principal fibre bundle, Publ. Dep. Math. Université de Lyon 1, 1/A, 1989.

[K4] —, The Chern-Weil homomorphism of regular Lie algebroids, Publ. Dep. Math. Université de Lyon 1, 1991.

[K5] - , A criterion for the Minimal Closedness of the Lie Subalgebra Corresponding to a Connected Nonclosed Lie Subgroup, Revista Matematica de la Universidad Complutense de Madrid 4, 1991.

[K6] - , Tangential Chern-Weil homomorphism, Proceedings of Geometric Study of Foliations, Tokyo, Nov. 1993, World Scientific, Singapore, 1994, pp. 324-344.

[K7] - Invariant cohomology of regular Lie algebroids, Proceedings of the VII International Colloquium on Differential Geometry, "Analysis and Geometry in Foliated Manifolds", Santiago de Compostela, Spain 26-30 July, 1994, World Scientific, Singapore 1995.

[K8] - Fibre integral in regular Lie algebroids, Proceedings of the Conference "Differential Geometry", Budapest, July 27-30, 1996, to appear.

[K9] - Some properties of regular Lie algebroids over foliated manifolds, in preparation.

[KU] A. Kumpera, An introduction to Lie groupoids, Nucleo de Estudos e Pasquisas Cientificas, Rio de Janeiro, 1971.

[M1] K. Mackenzie, Lie groupoids and Lie algebroids in Differential Geometry, London Mathematical Society Lecture Note Series 124, Cambridge, 1987.

[MR] L. Maxim-Raileanu, Cohomology of Lie algebroids, An. Sti. Univ. "Al. I. Cuza" Iasi. Sect. I a Mat. 22 (1976), 197-199.

[M-S] C. Moore and C. Schochet, Global Analysis on Foliated Spaces, Mathematical Sciences Research Institute publications 9, Springer-Verlag, 1988.

[MO1] P. Molino, Etude des feuilletages transversalement complets et applications, Ann. Sci. Ecole Norm. Sup. 10(3) (1977), 289-307.

[MO2] —, Riemannian foliations, Progress in Mathematics Vol. 73, Birkhäuser, Boston, Basel, 1988.

[N-V-Q] Ngô Van Quê, Du prolongement des espaces fibrés et des structures infinitésimales, Ann. Inst. Fourier (Grenoble) 17,1 (1967), 157-223.

[P1] J. Pradines, Théorie de Lie pour les groupoïdes différentiables. Calcul différentiel dans la catégorie des groupoïdes infinitésimaux, C. R. Acad. Sci. Ser. A-B, Paris 264 (1967), 245-248.

[P2] -, Théorie de Lie pour les groupoïdes différentiables, Atti Conv. Intern. Geom. 7 Diff. Bologna, 1967, Bologna-Amsterdam. 